\title{
Darstellung und optische Eigenschaften endständig substituierter Polyene mit Androstan als Spacer
}

\author{
Franz Effenberger* und Hartmut Strobel ${ }^{[1]}$ \\ Institut für Organische Chemie der Universität Stuttgart, \\ Pfaffenwaldring 55, W-7000 Stuttgart 80
}

Eingegangen am 1. Februar 1993

Key Words: Polyenes, conjugated / Bichromophores / Steroid (androstane) as spacer

\begin{abstract}
Synthesis and Optical Properties of Terminally Substituted Conjugated Polyenes with Androstane as Spacer
The conjugated polyenes 10, 11, 21, 22, and 26 with 9-anthryl and 2-tetraphenylporphyrinyl (TPP) terminal groups and an androstane unit incorporated into the polyene chain were synthesized by Wittig or Wittig-type olefinations. In most but not all cases the desired all-(E) isomers could be obtained from the $(E) /(Z)$-isomeric mixtures by chromatographic purification.

From the UV/Vis spectra it is concluded that in all cases the TPP group and the polyene chain are electronically separated, whereas the 9-anthryl residue and the connected dienyl moiety in 10 and 11 are electronically amalgamated. In 21, 22, and 26 a selective excitation of the anthryl group, the TPP moiety, and the polyene is possible.
\end{abstract}

In den letzten Jahren erlangten Untersuchungen zum Verständnis und zur Realisierung des Energietransfers auf molekularer Ebene zunehmende Bedeutung ${ }^{[2]}$. Neben grundlegenden Arbeiten zur Aufklärung des mechanistischen Ablaufs der Photosynthese ${ }^{[3]}$ hatten die Untersuchungen vor allem das Ziel, organische Verbindungen aufzufinden, die sich für einen Energie- oder Elektronentransfer und/oder eine Informationsspeicherung auf molekularer Basis eignen ${ }^{[4]}$. So konnte z. B. in konjugierten Polyenen unterschiedlicher Länge, die endständig jeweils mit einem 9-Anthryl- und Tetraphenylporphinyl-Rest substituiert sind, ein intramolekularer Energietransfer nachgewiesen werden ${ }^{[5]}$.

In weiteren Untersuchungen Donor-Acceptor-substituierter konjugierter Polyene wurden die Möglichkeiten einer Unterbrechung oder Minderung des intramolekularen Energietransfers durch den Einbau gesättigter Moleküle (Spacer) in das konjugierte Polyen ermittelt ${ }^{[6]}$. Als Spacer wurde dabei das weitgehend starre Bicyclo[2.2.2] octan gewählt, das in unterschiedliche Positionen des konjugierten Systems eingebaut wurde ${ }^{[6]}$. Aus den UV/Vis-Spektren der jeweiligen Verbindungen geht hervor, daß der intramolekulare Energietransfer in diesen Modellverbindungen durch den Einbau eines Bicyclo[2.2.2] octans als Spacer nicht unterbrochen wird ${ }^{[6,7]}$. Darüber hinaus wurde aus den Spektren eine deutliche Abhängigkeit des elektronischen Gesamtzustandes dieser Verbindungen von der Position des Spacers innerhalb der konjugierten Kette erkennbar ${ }^{[6,7]}$. Nur bei ausreichend großem Abstand zwischen den Endgruppen und dem Spacer sind die Einzelelemente des Moleküls elektronisch so weit getrennt, daß eine selektive Anregung der jeweiligen Molekülsegmente möglich ist ${ }^{[6,7]}$.

In der vorliegenden Publikation berichten wir über die Darstellung konjugierter Polyene, die als Endgruppen ebenfalls den 9-Anthryl- und Tetraphenylporphinyl-Rest besitzen, in die jedoch ein Steroidmolekül als Spacer eingebaut ist. Im Vergleich zu Bicyclo[2.2.2] octan, das mit einer Länge von ca. $3 \AA$ anzusetzen ist, wird mit einem Steroid als Spacer die Konjugation auf einer Länge von $9 \AA$ unterbrochen. Aus den oben angeführten Gründen ${ }^{[6,7]}$ kam der Synthese von Modellverbindungen, bei der das Steroid ebenfalls in unterschiedliche Positionen des konjugierten Polyens eingebaut ist, besondere Bedeutung zu.
Eine Diskussion der UV/Vis-Spektren dieser Modellverbindungen erfolgt in der vorliegenden Publikation nur in dem Rahmen, wie sie für die Charakterisierung der hergestellten Verbindungen unverzichtbar ist und wo darüber hinaus die spektroskopischen Befunde die Basis für die Synthese weiterer Modellverbindungen darstellen. Eine allgemeine Diskussion der theoretischen und physikalischen Grundlagen zum Mechanismus des Energietransfers in diesen Verbindungen wird an Hand der Ergebnisse umfangreicher spektroskopischer Untersuchungen ${ }^{[8 \mathrm{a}, \mathrm{b}]}$ in einer separaten Veröffentlichung erfolgen ${ }^{[8 c]}$.

Bichromophore Systeme mit Androstan als Spacer wurden erstmals von Keller und Dolby dargestellt und im Hinblick auf Energietransfererscheinungen untersucht ${ }^{[9]}$. In neuerer Zeit haben sich besonders Wu et al. ${ }^{[10]}$ und Tong et al. ${ }^{[11]}$ mit der Möglichkeit, Steroide als Spacer für Energietransferprozesse einzusetzen, beschäftigt. Vor allem zur Untersuchung von Long-range-Elektronentransferprozessen wurden Steroide als Spacer verwendet ${ }^{[12,13]}$.

Wie oben schon erwähnt, hängen elektronische Anregung und Energietransfereigenschaften Donor-Acceptor-substituierter Polyene mit Bicyclo[2.2.2] octan als Spacer von der Stellung des Spacers im Polyen $\mathrm{ab}^{[6,7]}$. Unter Berücksichtigung dieser Befunde haben wir zwei Strukturtypen DonorAcceptor-substituierter Polyene mit Androstan als Spacer synthetisiert und ihre UV/Vis-Spektren untersucht:

a) die Verbindungen $\mathbf{1 0}$ und 11, bei denen der Spacer in das konjugierte Polyen eingebaut ist und

b) die Verbindungen 21 und 22, bei denen der Spacer an einem Ende direkt mit dem Donor, dem 9-Anthryl-Rest, und am anderen Ende mit der Polyenkette verknüpft ist.

\section{Darstellung der Polyene 10 und 11}

Die Darstellung der Steroid-verbrückten AnthracenTPP-Polyene 10 und 11 ist in Schema 1 zusammengefaßt. 
Schema 1<smiles>CC12CCC3C(CCC4CC(O)CCC43C)C1CCC2=O</smiles>
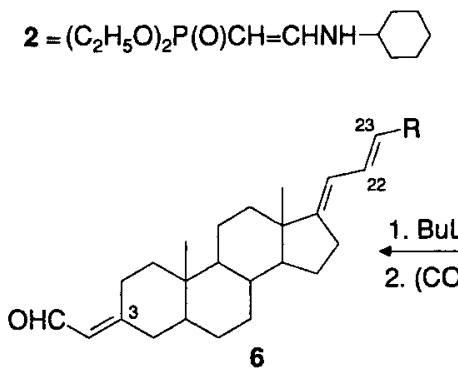

6

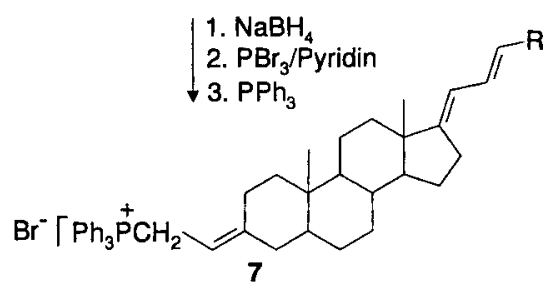

BuLi $\downarrow+8$ bzw. 9
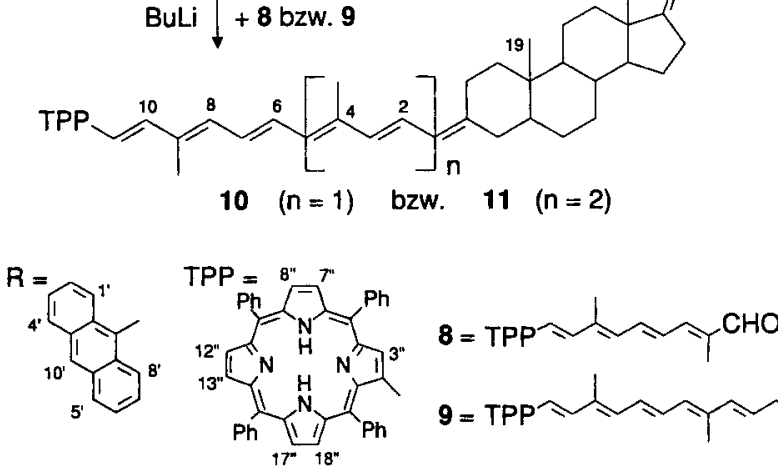

$$
9=T P P \curvearrowright \text { A }
$$

Die OH-Gruppe der Ausgangsverbindung 3 $\beta$-Hydroxy$5 \alpha$-androstan-17-on (epi-Androsteron) (1) wird zunächst als Tetrahydropyranylderivat ${ }^{[14]}$ geschützt. Durch Umsetzung mit einem Überschuß an [2-(Cyclohexylamino)vinyl]phosphonsäure-diethylester (2) ${ }^{[15]}$ und anschließende Hydrolyse des primär gebildeten Aldimins mit Oxalsäure wird der $\alpha, \beta$ ungesättigte Aldehyd 3 in 60proz. Ausbeute erhalten. Zur Gewinnung des Ketons 5 wird 3 zuerst in einer Wittig-Reaktion mit (Anthrylmethyl)phosphoniumbromid $4^{[16]}$ umgesetzt, anschließend die Tetrahydropyranyl-Schutzgruppe mit $p$-Toluolsulfonsäure in siedendem Ethanol abgespalten ${ }^{[17]}$ und zum Schluß der gebildete Alkohol in einer Oppenauer-Oxidation mit Cyclohexanon als Oxidationsmittel in Gegenwart von Aluminiumtriisopropylat in siedendem Toluol mit 92\% Ausbeute zum Keton 5 oxidiert.

Durch Umsetzung von 5 mit dem Phosphonat 2 und anschließende Hydrolyse konnte der $\alpha, \beta$-ungesättigte Aldehyd 6 mit 55\% Ausbeute bezogen auf 5 hergestellt werden. 6 liegt als Isomerengemisch sowohl bezüglich der Doppelbindung an Position 3 des Steroids als auch bezüglich der zwischen den C-Atomen 22 und 23 des Diens vor. Aufgrund unterschiedlicher Kopplungskonstanten können die vier möglichen Isomere ${ }^{1} \mathrm{H}-\mathrm{NMR}$-spektroskopisch nachgewiesen und zugeordnet werden.

Der Aldehyd 6 wird mit $\mathrm{NaBH}_{4}$ in Methanol/Dichlormethan in 87proz. Ausbeute zum entsprechenden Alkohol reduziert, der anschließend mit Phosphortribromid in Pyridin zum entsprechenden Bromderivat umgesetzt wird, das mit Triphenylphosphan in die Phosphonium-Verbindung 7 übergeführt wird. Durch Zugabe des Pyridins wird ein $\mathrm{S}_{\mathrm{N}} 2$ Verlauf der Bromierung begünstigt und gleichzeitig das Auftreten von freier Bromwasserstoff-Säure, die zu Nebenreaktionen führen würde, vermieden ${ }^{[18]}$.

Durch Wittig-Reaktion des Phosphonium-Salzes 7 mit den Tetraphenyl-21,23-dihydroporphyrinyl-Polyenalen 8 bzw. 9 konnten die gewünschten Zielmoleküle $\mathbf{1 0}$ und $\mathbf{1 1}$ dargestellt werden. Das TPP-Polyenal 8 ist bereits beschrieben ${ }^{[6,19]}$. Das TPP-Polyenal 9 haben wir durch Umsetzung von 8 mit (4,4-Dimethoxy-3-methyl-2-butenyl)triphenylphosphoniumchlorid (18) (s. Schema 2) in 66proz. Ausbeute bezogen auf das eingesetzte 8 erhalten.

Bei der Darstellung von 10 aus 7 und 8 wurde ein 30proz. Überschuß an TPP-Polyenal 8 eingesetzt, um 7 möglichst vollständig umzusetzen, da bei der wäßrigen Aufarbeitung nicht umgesetztes Phosphoniumsalz 7 zur entsprechenden unpolaren Methylverbindung hydrolysiert wird ${ }^{[20]}$, die nur durch mehrmalige Chromatographie von $\mathbf{1 0}$ abzutrennen ist. Verbindung 11 wurde dagegen mit einem Überschuß an 7 hergestellt. Die Verbindungen 10 und 11 wurden mit 14 bzw. 43\% Ausbeute, bezogen auf 7 bzw. 9, als $(E) /(Z)$-Isomerengemische erhalten. Bei 10 konnten nach mehrmaliger HPLC neben dem all-(E)-Isomer mit $98 \%$ Isomerenreinheit zwei $(Z)$-Isomere abgetrennt und ${ }^{1} \mathrm{H}$-NMR-spektroskopisch zugeordnet werden. Eine Trennung der Isomere von 11 war dagegen nicht möglich.

\section{UV/Vis-Spektrum von 10}

Im UV/Vis-Spektrum von Verbindung 10 (Abb. 1) ist die im Bereich von $\lambda=240-280 \mathrm{~nm}$ auftretende intensive Bande auf die Absorption des Anthryldienchromophors zurückzuführen ${ }^{[6]}$. Zusätzlich treten bei $300 \mathrm{~nm}$ Absorptionsbanden auf, die vermutlich einer Mischung von Ni-

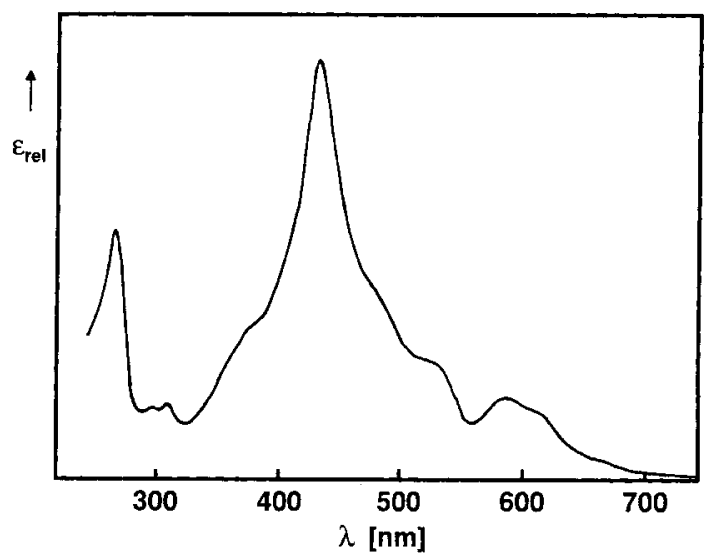

Abb. 1. UV/Vis-Absorptionsspektrum von $10\left(c=5.2 \cdot 10^{-6} \mathrm{M}\right)$ in Dichlormethan bei $25^{\circ} \mathrm{C}$ 
veaus beider Teilchromophore, nämlich dem des Anthracens und dem des Diens, zuzuordnen sind ${ }^{[8]}$. Der langwellige Bereich wird erwartungsgemäß von der Absorption des TPPRestes dominiert ${ }^{[6]}$. Im Bereich $\mathrm{ab} \lambda=340 \mathrm{~nm}$ ist die TPPPolyen-Absorption erkennbar. Die breite und wenig strukturierte Bande läßt auf eine starke Wechselwirkung zwischen Polyen und Porphyrin schließen. Das Spektrum läßt keine Wechselwirkungen zwischen den beiden durch den Steroid-Spacer getrennten Molekülteilen erkennen.

\section{Darstellung der 9-Anthryl-Steroid-TPP-Polyene 21 und 22}

Da sich, wie das UV/Vis-Spektrum von 10 zeigt, die Anthrylgruppe und der Dienteil zwischen Anthrylrest und Spacer elektronisch nicht getrennt anregen lassen, war es zur Klärung des Mechanismus des Energietransfers notwendig, Verbindungen zu synthetisieren, bei denen Anthracen direkt mit dem Steroid verknüpft ist. Die Synthese der Zielmoleküle 21 und 22 ist in Schema 2 zusammengefaßt.

Ausgangsverbindung für die Darstellung der Verbindungen 21 und 22 ist 3,17-Androstandion (13), das durch JonesOxidation $^{[21]}$ mit $\mathrm{CrO}_{3}$ leicht aus 1 zugänglich ist. Eine Anknüpfung des Anthracens an $\mathbf{1 3}$ ist aus sterischen Gründen nur in 3-Position möglich, was zu einer räumlichen Umkehrung des Steroid-Spacers im Vergleich zu 10 und 11 führt.

13 wurde mit metalliertem 9,10-Dihydroanthracen (12) in 27 proz. Ausbeute zum Alkohol 14 umgesetzt, wobei die sterisch gehinderte 17-Position nicht geschützt werden muß. Durch Erhitzen von 14 in Toluol mit katalytischen Mengen p-Toluolsulfonsäure haben wir (Dihydroanthryl)androstenon 15 mit 83\% Ausbeute erhalten, das erst in siedendem Xylol in Gegenwart molarer Mengen $p$-Toluolsulfonsäure zu 16 isomerisiert. Da unter diesen energischen Bedingungen die elektrophile Aromatensubstitution reversibel wird, was über die Protonierung des Anthracens in 9-Position zur Abspaltung des Androstenyl-Restes führt, wurde die Isomerisierung schon bei einem Edukt-Produkt-Verhältnis von 35:65 abgebrochen. Nach Mitteldruckchromatographie konnte 16 so mit etwa 20\% Ausbeute, ausgehend von 15, isoliert werden. Die Struktur von 16 wurde NMR-spektroskopisch ermittelt und zeigt $\boldsymbol{\beta}$-Konfiguration an $\mathrm{C}-3$ des Steroids.

Durch Wittig-Reaktion von 16 mit 2 und anschließende Hydrolyse mit Oxalsäure wurde das Anthrylpregnenal 17 mit 69\% Ausbeute bezogen auf 16 isoliert. Aus 17 haben wir durch Umsetzung mit dem Phosphoniumsalz 18 und nachfolgende Spaltung des Acetals das Trienal 19 aufgebaut, das als $(E) /(Z)$-Isomerengemisch im Verhältnis von ca. 1:1 vorliegt. Das all-(E)-Isomer von 19 wird nach Abtrennung durch Mitteldruckchromatographie mit 38\% Ausbeute bezogen auf 17 erhalten. Das zum weiteren Aufbau der Verbindungen 21 und $\mathbf{2 2}$ erforderliche Allylphosphonium-Salz 20 wurde ausgehend vom leicht zugänglichen TPP-CHO ${ }^{[22]}$ hergestellt (s. Exp. Teil): Der durch Umsetzung mit [(Ethoxycarbonyl)methyl]phosphonsäure-diethylester ${ }^{[23]} \mathrm{er}-$ haltene TPP-Acrylsäure-ethylester ${ }^{[24]}$ wird mit Diisobutylaluminiumhydrid (DIBAL) in THF zum entsprechenden
Allyl-Alkohol reduziert, der anschließend mit Phosphortribromid in $\mathrm{DMF}^{[25]}$ bromiert wird. Da sich das äußerst reaktive Bromid bei der chromatographischen Reinigung zersetzte, wurde das Rohprodukt weiter mit Triphenylphosphan in siedendem Toluol zu 20 umgesetzt.

Schema 2

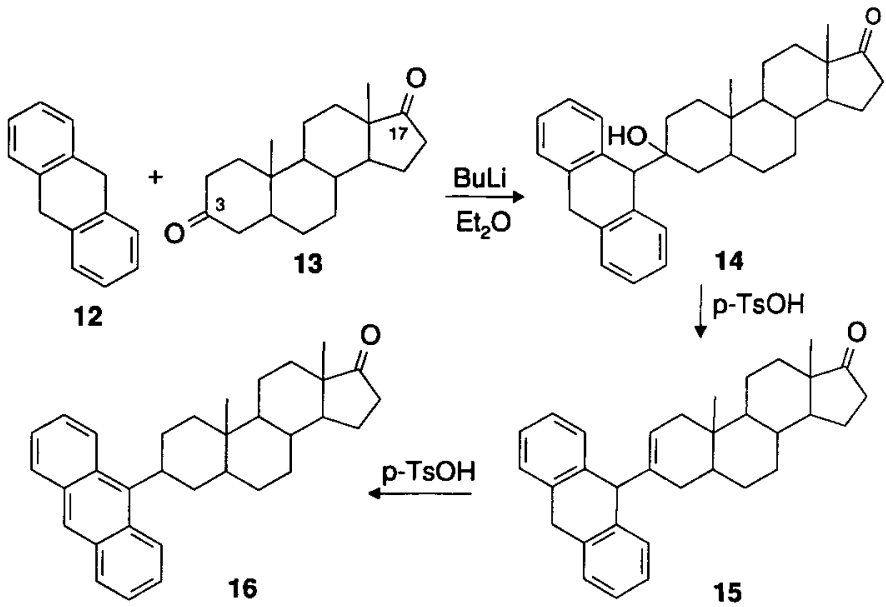

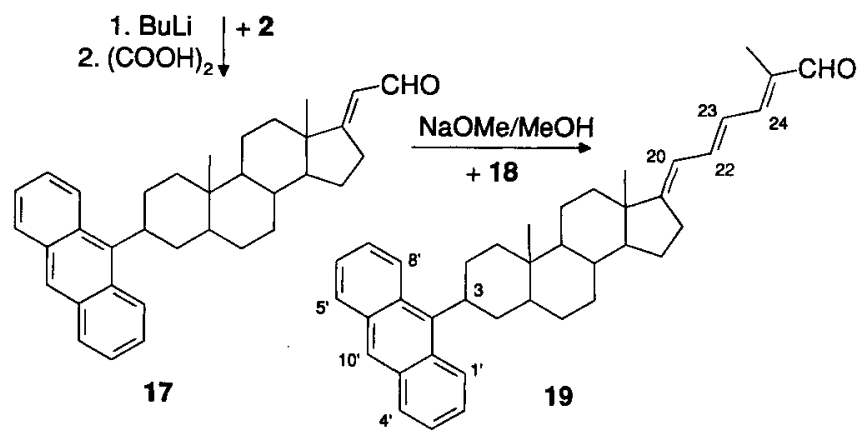<smiles>COC(OC)/C(C)=C/CP[13CH2][13CH3]</smiles><smiles>CO[N+](C)(O)c1ccccc1</smiles>

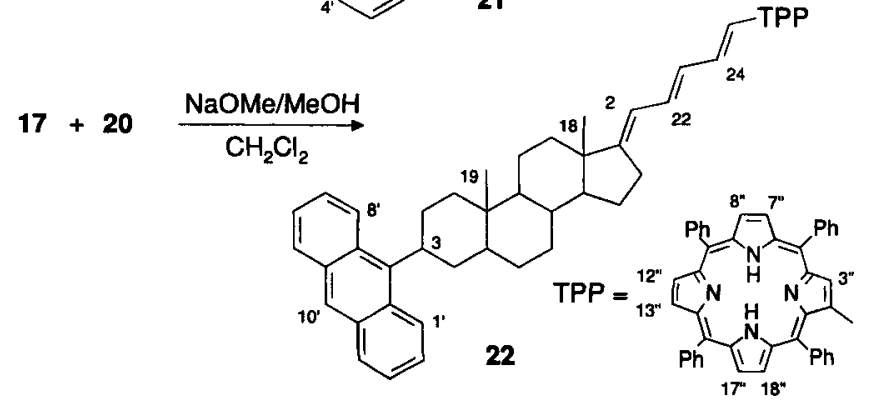


all-(E)-19 wurde mit dem TPP-Phosphoniumsalz $20 \mathrm{zu}$ der Anthracen-Steroid-verknüpften Zielverbindung $\mathbf{2 1}$ gekoppelt. Nach Chromatographie an Kieselgel haben wir $\mathbf{2 1}$ als $(E) /(Z)$-Isomerengemisch mit einem Anteil von etwa $80 \%$ des all-(E)-Isomeren erhalten, das durch Mitteldruckchromatographie gereinigt und mit $32 \%$ Ausbeute und einem all-(E)-Anteil von $98 \%$ isoliert wurde. Der all-(E)-Anteil konnte durch weitere Anreicherung auf $99 \%$ gesteigert werden.

Entsprechend wurde 17 mit einem Überschuß an Allylphosphoniumsalz 20 in 19proz. Ausbeute zur Zielverbindung 22 umgesetzt. Das all-(E)-Isomer von 22 konnte mitteldruckchromatographisch aus dem erhaltenen $(E) /(Z)$-Isomerengemisch abgetrennt und mit einem all- $(E)$-Anteil von $97 \%$ isoliert werden.

\section{Darstellung des Anthracen-TPP-Polyens 26}

Die Verkürzung der Polyen-Kette zwischen Steroid-Spacer und TPP-Endgruppe sollte eine Quantifizierung der Energietransferprozesse ermöglichen. Das Trien wurde deshalb gewählt, weil die Absorption energetisch genau zwischen den $S_{0} \rightarrow S_{3}$ - und $S_{0} \rightarrow S_{1}$-Absorptionsbanden des Anthrylrestes erfolgt. Aus diesem Grund wurde Verbindung 22 dargestellt. Um nun das durch den Steroid-Spacer unterbrochene Donor-Acceptor-substituierte Polyen 22 mit den früher untersuchten Modellverbindungen ${ }^{[5]}$ vergleichen zu können, haben wir noch das Anthryl-TPP-Polyen 26 mit drei konjugierten Doppelbindungen zwischen den beiden Endgruppen hergestellt (Schema 3).

Schema 3<smiles>O=Cc1c2ccccc2cc2ccccc12</smiles>

23

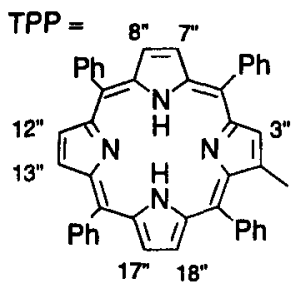<smiles>CC(/C=C/[In](C)C)=C\C=C\c1c2ccccc2cc2ccccc12</smiles>

Die Umsetzung von Anthracen-9-carbaldehyd (23) mit 18 zum Dienal 24 ist bereits beschrieben worden ${ }^{[26]}$. Das TPPPhosphoniumsalz 25 konnte durch Reaktion des leicht zugänglichen Hydroxymethyl-TPP ${ }^{[22 a]}$ - analog der Darstellung von 20 - mit Phosphortribromid in DMF und anschließende Umsetzung mit Triphenylphosphan in sieden- dem Toluol mit 39\% Ausbeute bezogen auf HydroxymethylTPP erhalten werden.

Bei der Knüpfungsreaktion von 24 mit 25 wurde 24 in 5 fachem ÜberschuB mit Natriummethanolat in Methanol vorgelegt und eine Lösung des TPP-Phosphoniumsalzes 25 in Methanol zugetropft. Aus dem erhaltenen Isomerengemisch wurde das all-(E)-Isomer von 26 durch Mitteldruckchromatographie und Umkristallisation in 30proz. Ausbeute mit einer Isomerenreinheit von $99.6 \%$ angereichert.

\section{UV/Vis-Spektren von 22 und 26}

In $\mathrm{Abb} .2$ sind die Absorptionsspektren der Verbindungen 22 und 26 wiedergegeben. Dabei zeigen sich im Bereich von $\lambda=200-400 \mathrm{~nm}$ deutliche Unterschiede zwischen beiden Verbindungen. So ist bei $\mathbf{2 2}$ die Absorptionsbande des $\mathrm{S}_{0} \rightarrow \mathrm{S}_{3}$-Überganges von Anthracen bei $\lambda=260 \mathrm{~nm}$ wegen der direkten Anknüpfung des Steroid-Spacers deutlich schärfer als im konjugierten Molekül 26. Die Bande zwischen $\lambda=280$ und $340 \mathrm{~nm}$ bei 22 kann aufgrund der Lage dem Trien zugeordnet werden. Die daneben auftretende niederenergetische Bande entspricht der charakteristischen $\mathrm{S}_{0} \rightarrow \mathrm{S}_{1}$-Schwingungsstruktur der Anthrylabsorption. Beide Banden fehlen im Spektrum von Verbindung 26. Die Absorption des TPP-Restes oberhalb von $\lambda=400 \mathrm{~nm}$ ist dagegen bei beiden Verbindungen identisch. Die direkte Anbindung des Anthracens an das konjugierte System hat bei 26 mit drei Doppelbindungen zwischen Donor und Acceptor nur geringen Einfluß auf die Absorption des PorphyrinRestes.

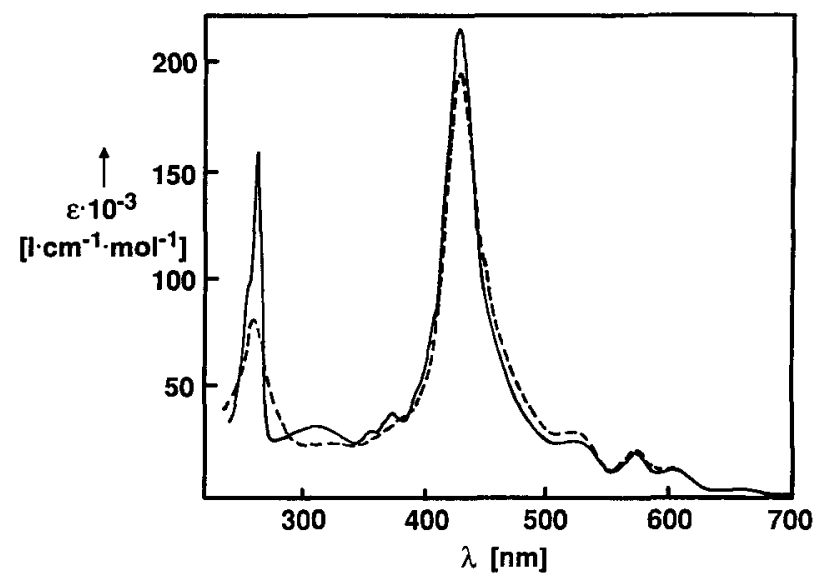

Abb. 2. UV/Vis-Absorptionsspektren von $22(\longrightarrow)\left(c=6.1 \cdot 10^{-6}\right.$ M) und $26(---)\left(c=6.4 \cdot 10^{-6} \mathrm{M}\right)$ in Dichlormethan bei $25^{\circ} \mathrm{C}$

Das UV/Vis-Absorptionsspektrum von 21 stimmt in den Anthracen- sowie den TPP-Absorptionsbanden nahezu mit 22 überein und läßt auf eine praktisch vollständige Unterbrechung der elektronischen Wechselwirkungen durch den Spacer schließen ${ }^{[\mathrm{sc}]}$.

Wir danken der Deutschen Forschungsgemeinschaft (SFB 329) und dem Fonds der Chemischen Industrie für die finanzielle Unterstützung dieser Arbeit. 


\section{Experimenteller Teil}

${ }^{1}$ H-NMR: Bruker WP 80, AC $250 \mathrm{~F}$ sowie CXP 300, TMS als interner Standard. - UV/Vis: Perkin-Elmer Lambda 7. - HPLC: Spektrophotometer Uvikon 720 LC (Kontron), Pumpe Milton Roy Constametric III, Säulen $(200 \times 4 \mathrm{~mm})$ mit Nucleosil Si $100,5 \mathrm{CN}$, $5 \mathrm{NO}_{2}$ (Macherey-Nagel). - MPLC: Säulen $(50 \times 4 \mathrm{~cm}) \mathrm{mit}$ LiChroprep Si 60, Korngröße 0.015-0.025 mm (Merck), Nucleosil $1525 \mathrm{CN}$, Korngröße 0.015-0.025 mm (Macherey-Nagel) sowie Säule $(35 \times 3 \mathrm{~cm})$ mit Nucleosil $1525 \mathrm{NO}_{2}$, Korngröße $0.010 \mathrm{~mm}$ (Macherey-Nagel). - Präparative Säulenchromatographie: Glassäulen verschiedener Größe mit Kieselgel, Korngröße 0.032-0.063 $\mathrm{mm}$ sowie Aluminiumoxid, Aktivitätsstufe III. - Alle Lösungsmittel wurden gereinigt und destilliert eingesetzt.

[2-(Cyclohexylamino) vinyl]phosphonsäure-diethylester (2): Nach Lit. $^{[15]}$.

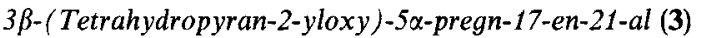

a) $3 \beta$-(Tetrahydropyran-2-yloxy)-5 $\alpha$-androstan-17-on: Nach Lit. ${ }^{[14]}$; aus $30 \mathrm{~g}(0.10 \mathrm{~mol}) 1,84.1 \mathrm{~g}(1.0 \mathrm{~mol}) 3,4$-Dihydro-2H-pyran und $5 \mathrm{mg} p$-Toluolsulfonsäure in $900 \mathrm{ml}$ wasserfreiem Diethylether;

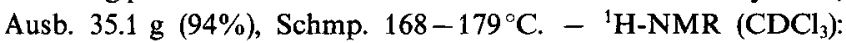
$\delta=0.50-2.70\left(\mathrm{~m}\right.$, Steroid-H), darin $0.85\left(\mathrm{~s}, 6 \mathrm{H}, \mathrm{CH}_{3}-18,-19\right)$, $3.30-4.07$ (m, 3 H, 6- $\mathrm{H}_{\text {pyranyl }}$, Ster-3-H), 4.70 (m, $\left.1 \mathrm{H}, 2-\mathrm{H}_{\text {Pyranyl }}\right)$. $\mathrm{C}_{24} \mathrm{H}_{38} \mathrm{O}_{3}$ (374.6): ber. C 76.96, $\mathrm{H} 10.23$; gef. C 76.90, H 10.34.

b) Zu $2.19 \mathrm{~g}(8.38 \mathrm{mmol}) 2$ in $15 \mathrm{ml}$ Tetrahydrofuran (THF) tropft man bei $0^{\circ} \mathrm{C} 5.2 \mathrm{ml}(8.32 \mathrm{mmol})$ einer $1.6 \mathrm{M}$ Lösung von $\mathrm{BuLi}$ in Hexan, rührt 20 min nach, spritzt anschließend eine Lösung von $0.63 \mathrm{~g}(1.68 \mathrm{mmol}) 3 \beta$-(Tetrahydropyran-2-yloxy)-5 $\alpha$-androstan-17on in $25 \mathrm{ml}$ THF zu und rührt $15 \mathrm{~h}$ bei Raumtemp. Das Reaktionsgemisch wird auf $30 \mathrm{ml}$ eiskaltes Wasser gegossen und viermal mit Diethylether extrahiert. Die wäßrige Phase wird mit Natriumchlorid gesättigt und nochmals mit Diethylether extrahiert. Die vereinigten Extrakte werden zweimal mit ges. NatriumchloridLösung gewaschen, eingeengt, und der Rückstand wird in $20 \mathrm{ml}$ Benzol aufgenommen. Man setzt $60 \mathrm{ml} 1 \mathrm{proz}$. Oxalsäure zu, rührt $16 \mathrm{~h}$ bei Raumtemp., extrahiert das Reaktionsgemisch mit Dichlormethan, trocknet mit Natriumsulfat und engt ein. Der Rückstand wird über eine 5-cm-Schicht von Aluminiumoxid gegeben. Das Produkt wird aus Dichlormethan mit Hexan ausgefällt; Ausb. $0.40 \mathrm{~g}$ (59\%) 3, Schmp. $146-148^{\circ} \mathrm{C} .-{ }^{1} \mathrm{H}-\mathrm{NMR}\left(\mathrm{CDCl}_{3}\right): \delta=0.64-1.90$ $(\mathrm{m}$, Steroid- $\mathrm{H})$, darin 0.84 und 0.85 (je s, $\left.6 \mathrm{H}, \mathrm{CH}_{3}-18,-19\right)$, 2.76-2.97 (m, 2H, Ster-16-H), 3.45-3.64 (m, 2H, 6- $\left.\mathrm{H}_{\text {Pyrany })}\right)$, $3.89-3.96\left(\mathrm{~m}, 1 \mathrm{H}\right.$, Ster-3-H), 4.68-4.72 (m, $\left.1 \mathrm{H}, 2-\mathrm{H}_{\text {Pyranyl }}\right), 5.74$ (td, $\left.{ }^{3} J_{20, \mathrm{CHO}}=8.0,{ }^{4} J_{20,16}=2.3 \mathrm{~Hz}, 1 \mathrm{H}, 20-\mathrm{H}\right), 9.86$ (d, $\left.1 \mathrm{H}, \mathrm{CHO}\right)$. $\mathrm{C}_{26} \mathrm{H}_{40} \mathrm{O}_{3}$ (400.6): ber. C 77.95, H 10.06; gef. C 77.73, H 10.19 .

$$
\begin{aligned}
& \text { [(9-Anthryl)methyl]triphenylphosphoniumbromid (4): Nach } \\
& \text { Lit. }^{[16]} \text {. }
\end{aligned}
$$

\section{3-(9-Anthryl)-5 $\alpha$-21,24-dinorchola-17,22-dien-3-on (5)}

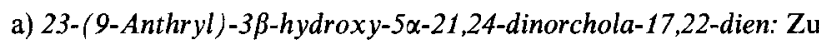
$15.99 \mathrm{~g} \mathrm{(29.97 \textrm {mmol } )} 4$ in $400 \mathrm{ml}$ wasserfreiem Diethylether spritzt man $18.73 \mathrm{ml}$ (29.97 mmol) einer 1.6 $\mathrm{M}$ Lösung von BuLi in Hexan, rührt $1 \mathrm{~h}$ nach und setzt zur Zerstörung von überschüssigem $\mathrm{BuLi}$ $1 \mathrm{ml}$ Dichlormethan zu. Dann spritzt man die Lösung von $4.0 \mathrm{~g}$ (9.99 mmol) 3 in $250 \mathrm{ml}$ Dichlormethan zu, rührt $1 \mathrm{~h}$ nach, engt das Reaktionsgemisch ein und nimmt den Rückstand in $500 \mathrm{ml}$ Ethanol auf. Man setzt $5 \mathrm{mg} p$-Toluolsulfonsäure zu, erhitzt $10 \mathrm{~min}$ unter Rückfluß, engt i.Vak. ein und chromatographiert den Rückstand an Kieselgel (Säule $5 \times 20 \mathrm{~cm}$ ) mit Dichlormethan/Ethylacetat (7:1). Das Produkt wird aus Diethylether/Hexan umkristallisiert und i. Hochvak. bei $70^{\circ} \mathrm{C}$ getrocknet; Ausb. $3.1 \mathrm{~g}(63 \%)$, Schmp. $126-131^{\circ} \mathrm{C}$. $-{ }^{1} \mathrm{H}$-NMR $\left(\mathrm{CDCl}_{3}\right): \delta=0.60-2.0(\mathrm{~m}$, Steroid-H), $2.37-2.62(\mathrm{~m}, 2 \mathrm{H}$, Ster-16-H), 3.54-3.65 (m, $1 \mathrm{H}$, Ster-3-
H), $6.15\left(\mathrm{~d}, J_{20,22}=10.9 \mathrm{~Hz}, 1 \mathrm{H}, 20-\mathrm{H}\right), 6.72\left(\mathrm{dd}, J_{22,23}=15.9 \mathrm{~Hz}\right.$, $1 \mathrm{H}, 22-\mathrm{H}), 7.22$ (d, $1 \mathrm{H}, 23-\mathrm{H}), 7.37-7.52$ (m, 4H, 2'-, 3'-, 6'-, 7'H), $7.83-8.02\left(\mathrm{~m}, 2 \mathrm{H}, 4^{\prime}-, 5^{\prime}-\mathrm{H}\right), 8.33-8.46\left(\mathrm{~m}, 3 \mathrm{H}, 1^{\prime}-, 8^{\prime}-, 10^{\prime}-\mathrm{H}\right)$. - $\mathrm{C}_{36} \mathrm{H}_{42} \mathrm{O}$ (490.7): ber. C 88.11, H 8.63; gef. C 88.24, H 8.78.

Daneben werden ca. $13 \%$ des $(Z)$-Isomeren ${ }^{1} \mathrm{H}$-NMR-spektroskopisch nachgewiesen.

b) In einem Zweihalskolben mit Tropftrichter und Rückflußkühler mit Blasenzähler und Sicapent-Trockenrohr erhitzt man unter $\mathrm{N}_{2}$ und Lichtausschluß $0.15 \mathrm{~g}$ (0.31 mmol) 23-(9-Anthryl)-3ß-hydroxy-5 $\alpha$-21,24-dinorchola-17,22-dien in $45 \mathrm{ml}$ Toluol und $3 \mathrm{ml}$ $(28.95 \mathrm{mmol})$ Cyclohexanon zum Sieden und destilliert langsam 10 $\mathrm{ml}$ Toluol ab. Innerhalb $45 \mathrm{~min}$ tropft man eine Lösung von $0.30 \mathrm{~g}$ (1.47 mmol) Aluminiumtriisopropylat in $15 \mathrm{ml}$ Toluol unter weiterem Abdestillieren von Toluol zu. Man versetzt das abgekühlte Reaktionsgemisch mit $15 \mathrm{ml}$ kalt ges. Kaliumnatriumtartrat-Lösung und entfernt überschüssiges Cyclohexanon durch Wasserdampfdestillation. Der Rückstand wird mit Dichlormethan extrahiert, die vereinigten Extrakte werden mit Wasser gewaschen, mit Natriumsulfat getrocknet und eingeengt. Der Rückstand wird an Kieselgel mit Dichlormethan chromatographiert; Ausb. $0.14 \mathrm{~g} \mathrm{(92 \% )} \mathrm{5,}$ Schmp. $225-227^{\circ} \mathrm{C}$. $-{ }^{1} \mathrm{H}-\mathrm{NMR}\left(\mathrm{CDCl}_{3}\right): \delta=0.78-2.59$ (m, Steroid-H), darin 0.92 und 1.06 (je s, $\left.6 \mathrm{H}, \mathrm{CH}_{3}-18,-19\right), 6.16$ (d, $\left.{ }^{4} J_{20,16}=2.2,{ }^{3} J_{20,22}=10.9 \mathrm{~Hz}, 1 \mathrm{H}, 20-\mathrm{H}\right), 6.72\left(\mathrm{dd}, J_{22,23}=16.0 \mathrm{~Hz}\right.$, $1 \mathrm{H}, 22-\mathrm{H}), 7.23$ (d, $1 \mathrm{H}, 23-\mathrm{H}), 7.43-7.53\left(\mathrm{~m}, 4 \mathrm{H}, 2^{\prime}-, 3^{\prime}-, 6^{\prime}-, 7^{\prime}-\right.$ H), 7.96-8.01 (m, 2H, 4'-, 5'-H), $8.29-8.41\left(\mathrm{~m}, 3 \mathrm{H}, 1^{\prime}-, 8^{\prime}-, 0^{\prime}-\mathrm{H}\right)$. $-\mathrm{C}_{36} \mathrm{H}_{40} \mathrm{O}$ (488.7): ber. C 88.48, H 8.25; gef. C 88.27, H 8.39.

[23-(9-Anthryl)-5 $\alpha$-21,24-dinorchola-17,22-dien-3-yliden]acetaldehyd (6): $\mathrm{Zu} 0.72 \mathrm{~g}(2.75 \mathrm{mmol}) 2$ in $10 \mathrm{ml}$ THF tropft man bei $0^{\circ} \mathrm{C} 1.72 \mathrm{ml}$ (2.75 mmol) einer $1.6 \mathrm{M}$ Lösung von BuLi in Hexan, rührt $20 \mathrm{~min}$ nach, spritzt eine Lösung von $0.45 \mathrm{~g}$ (0.92 mmol) 5 in $20 \mathrm{ml}$ THF zu und rührt $4 \mathrm{~h}$ bei Raumtemp. Das Reaktionsgemisch wird auf eiskaltes Wasser gegossen und mit Diethylether extrahiert. Die vereinigten Extrakte werden mit ges. Natriumchlorid-Lösung gewaschen und eingeengt. Man nimmt den Rückstand in Benzol auf, gibt $40 \mathrm{ml} 1$ proz. Oxalsäure zu, und nach 8 stdg. Rühren bei Raumtemp. wird das Reaktionsgemisch mit Dichlormethan extrahiert. Die vereinigten Extrakte werden mit Natriumsulfat getrocknet und eingeengt. Der Rückstand wird an Kieselgel mit Dichlormethan chromatographiert und das Produkt aus Hexan/Ethylacetat kristallisiert; Ausb. $0.26 \mathrm{~g}(55 \%)$, Schmp. $213-216^{\circ} \mathrm{C} .-{ }^{1} \mathrm{H}$ NMR $\left(\mathrm{CDCl}_{3}\right): \delta=0.61-3.29(\mathrm{~m}$, Steroid-H), darin 0.90 und 0.99 (je s, $\left.6 \mathrm{H}, \mathrm{CH}_{3}-18,-19\right), 5.80-5.84(\mathrm{~m}, 1 \mathrm{H}, 1-\mathrm{H}), 6.15\left(\mathrm{~d}, J_{20,22}=10.9\right.$ $\mathrm{Hz}, 1 \mathrm{H}, 20-\mathrm{H}$ ), 6.72 (dd, $\left.J_{22,23}=15.8 \mathrm{~Hz}, 1 \mathrm{H}, 22-\mathrm{H}\right), 7.23(\mathrm{~d}, 1 \mathrm{H}$, 23-H), $7.42-7.49\left(\mathrm{~m}, 4 \mathrm{H}, 2^{\prime}-, 3^{\prime}-, 6^{\prime}-, 7^{\prime}-\mathrm{H}\right), 7.96-8.02(\mathrm{~m}, 2 \mathrm{H}$, $\left.4^{\prime}-, 5^{\prime}-\mathrm{H}\right), 8.33-8.47\left(\mathrm{~m}, 3 \mathrm{H}, 1^{\prime}-, 8^{\prime}-, 0^{\prime}-\mathrm{H}\right), 10.02$ und 10.03 (d, $\left.J_{1, \text { Сно }}=8.3 \mathrm{~Hz}, 1 \mathrm{H}, \mathrm{CHO}\right) .-\mathrm{C}_{38} \mathrm{H}_{42} \mathrm{O}(514.7)$ : ber. C 88.67, $\mathrm{H} 8.22$; gef. C 88.22, H 8.36.

Daneben wurden ca. $10 \%$ des $(Z)$-Isomeren ${ }^{1} H$-NMR-spektroskopisch nachgewiesen.

[2-[23-(9-Anthryl)-5 $\alpha$-21,24-dinorchola-17,22-dien-3-yliden]ethyl Jtriphenylphosphoniumbromid (7)

a) 23-(9-Anthryl)-3-(2-bromethyliden)-5 $\alpha$-21,24-dinorchola-17,22dien: In einen Zweihalskolben mit Septum und Rückflußkühler mit Sicapent-Trockenrohr und Blasenzähler gibt man bei $-10^{\circ} \mathrm{C}$ unter $\mathrm{N}_{2}$ zu $1.46 \mathrm{~g}(2.83 \mathrm{mmol}) 6$ in $70 \mathrm{ml}$ Dichlormethan $/ 0.5 \%$ Pyridin innerhalb $5 \mathrm{~min} 0.78 \mathrm{~g}$ ( $2.88 \mathrm{mmol})$ Phosphortribromid in $10 \mathrm{ml}$ Dichlormethan $/ 0.5 \%$ Pyridin, rührt $5 \mathrm{~min}$ bei $-10^{\circ} \mathrm{C}$ und gießt das Reaktionsgemisch nach 2 stdg. Rühren bei Raumtemp. (Reaktion dünnschichtchromatographisch verfolgt) auf $80 \mathrm{ml}$ eiskaltes Wasser. Die organische Phase wird mit Wasser neutral gewaschen, mit Natriumsulfat getrocknet und eingeengt; Rohausb. 
$1.96 \mathrm{~g}$, davon werden $0.96 \mathrm{~g}$ an geglühtem Kieselgel mit Diethylether chromatographiert; Ausb. $0.61 \mathrm{~g}(63 \%)$, Schmp. $115-117^{\circ} \mathrm{C}$. $-{ }^{1} \mathrm{H}-\mathrm{NMR}\left(\mathrm{CDCl}_{3}\right): \delta=0.55-2.60(\mathrm{~m}$, Steroid-H), darin 0.89 und 0.93 (je s, $\left.6 \mathrm{H}, \mathrm{CH}_{3}-18,-19\right), 3.99-4.06\left(\mathrm{~m}, 2 \mathrm{H}, \mathrm{CH}_{2} \mathrm{P}\right), 5.40-5.50$ $(\mathrm{m}, 1 \mathrm{H}, 1-\mathrm{H}), 6.15\left(\mathrm{~d}, J_{20,22}=10.9 \mathrm{~Hz}, 1 \mathrm{H}, 20-\mathrm{H}\right), 6.72(\mathrm{dd}$, $\left.J_{22,23}=15.9 \mathrm{~Hz}, 1 \mathrm{H}, 22-\mathrm{H}\right), 7.22(\mathrm{~d}, 1 \mathrm{H}, 23-\mathrm{H}), 7.42-7.48(\mathrm{~m}, 4 \mathrm{H}$, $\left.2^{\prime}-, 3^{\prime}-, 6^{\prime}-, 7^{\prime}-\mathrm{H}\right), 7.96-8.02\left(\mathrm{~m}, 2 \mathrm{H}, 4^{\prime}-, 5^{\prime}-\mathrm{H}\right), 8.34-8.39(\mathrm{~m}, 3 \mathrm{H}$, $\left.1^{\prime}-, 8^{\prime}-, 10^{\prime}-\mathrm{H}\right)$. - Das restliche Produkt wird ohne Reinigung weiter umgesetzt.

b) $\mathrm{Zu} 1.0 \mathrm{~g}$ (1.73 mmol) 23-(9-Anthryl)-3-(2-bromethyliden $)-5 \alpha$ 21,24-dinorchola-17,22-dien (Rohprodukt) in $30 \mathrm{ml}$ Toluol gibt man $0.45 \mathrm{~g}(1.72 \mathrm{mmol})$ Triphenylphosphan und erhitzt $24 \mathrm{~h}$ unter Rückfluß. Man gibt $30 \mathrm{ml}$ Diethylether zu, saugt den ausgefallenen Feststoff ab, kristallisiert zweimal aus Dichlormethan/Diethylether um und trocknet das Produkt bei $60^{\circ} \mathrm{C}$ i. Hochvak. über Sicapent und

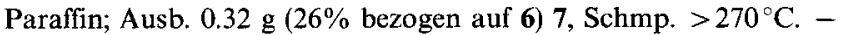
${ }^{1} \mathrm{H}-\mathrm{NMR}\left(\mathrm{CDCl}_{3}\right): \delta=0.70$ und 0.82 (je s, $6 \mathrm{H}, \mathrm{CH}_{3}-18,-19$ ), 3.42-3.70 (m, 2H, $\left.\mathrm{CH}_{2} \mathrm{P}\right), 5.01-5.43(\mathrm{~m}, 1 \mathrm{H}, 1-\mathrm{H}), 6.14(\mathrm{~d}$, $\left.J_{20,22}=11.0 \mathrm{~Hz}, 1 \mathrm{H}, 20-\mathrm{H}\right), 6.71\left(\mathrm{dd}, J_{22,23}=15.8 \mathrm{~Hz}, 1 \mathrm{H}, 22-\mathrm{H}\right)$, $7.20(\mathrm{~d}, 1 \mathrm{H}, 23-\mathrm{H}), 7.41-7.44\left(\mathrm{~m}, 4 \mathrm{H}, 2^{\prime}-, 3^{\prime}-, 6^{\prime}-, 7^{\prime}-\mathrm{H}\right), 7.71-7.78$ (m, 15 H, $\left.\mathrm{H}_{\text {Pheny }}\right), 7.94-7.97\left(\mathrm{~m}, 2 \mathrm{H}, 4^{\prime}-, 5^{\prime}-\mathrm{H}\right), 8.25-8.40(\mathrm{~m}, 3 \mathrm{H}$, $\left.1^{\prime}-, 8^{\prime}-, 10^{\prime}-\mathrm{H}\right)$.

Daneben wurden ca. $30 \%$ des $(Z)$-Isomeren 'H-NMR-spektroskopisch nachgewiesen.

9-(21,23-Dihydro-5,10,15,20-tetraphenylporphyrin-2-yl)-2,7-dimethyl-2,4,6,8-nonatetraenal (8): Nach Lit. ${ }^{[19]}$.

13-(21,23-Dihydro-5,10,15,20-tetraphenylporphyrin-2-yl)-2,6,11trimethyl-2,4,6,8,10,12-tridecahexaenal (9): Unter $\mathrm{N}_{2}$ gibt man unter Rühren zu $0.15 \mathrm{~g}(0.19 \mathrm{mmol}) 8$ und $1.16 \mathrm{ml}(1.16 \mathrm{mmol})$ einer $1 \mathrm{M}$ Lösung von Natriummethanolat in Methanol in einem Zweihalskolben mit Septum und Rückflußkühler mit Blasenzähier und Sicapent-Trockenrohr innerhalb $1 \mathrm{~h} 1.13 \mathrm{ml}(1.16 \mathrm{mmol})$ einer $1.03 \mathrm{M}$ Lösung von 18 in Methanol. Nach Beendigung der Reaktion (HPLC-Kontrolle) wird das Gemisch zweimal mit Wasser gewaschen, mit Natriumsulfat getrocknet und eingeengt. Der Rückstand wird zweimal an Kieselgel mit Dichlormethan chromatographiert, das Produkt aus Dichlormethan/Hexan umkristallisiert und bei $50^{\circ} \mathrm{C}$ i. Hochvak. über Sicapent und Paraffin getrocknet; Ausb. $0.105 \mathrm{~g}(66 \%)$, Schmp. $271{ }^{\circ} \mathrm{C}$. $-{ }^{1} \mathrm{H}-\mathrm{NMR}\left(\mathrm{CDCl}_{3}\right): \delta=1.70(\mathrm{~s}$, $\left.3 \mathrm{H}, 6-\mathrm{CH}_{3}\right), 1.91\left(\mathrm{~s}, 3 \mathrm{H}, 11-\mathrm{CH}_{3}\right), 2.02\left(\mathrm{~s}, 3 \mathrm{H}, 2-\mathrm{CH}_{3}\right), 6.44(\mathrm{~d}$, $J=10.7 \mathrm{~Hz}, 1 \mathrm{H}, 7-\mathrm{H}), 6.47(\mathrm{~d}, 1 \mathrm{H}, 10-\mathrm{H}), 6.56\left(\mathrm{~d}, J_{12,13}=15.6 \mathrm{~Hz}\right.$, $1 \mathrm{H}, 12-\mathrm{H}), 6.61-6.90(\mathrm{~m}, 4 \mathrm{H}, 4-, 5-, 8-, 9-\mathrm{H}), 6.94\left(\mathrm{~d}, J_{3,4}=9.7 \mathrm{~Hz}\right.$, $1 \mathrm{H}, 3-\mathrm{H}), 7.13$ (d, $1 \mathrm{H}, 13-\mathrm{H}), 7.71-7.83\left(\mathrm{~m}, 12 \mathrm{H}, m-, p-\mathrm{H}_{\text {Phenyl }}\right)$, $8.09-8.24\left(\mathrm{~m}, 8 \mathrm{H}, o-\mathrm{H}_{\text {Phenyl }}\right), 8.65-8.83\left(\mathrm{~m}, 6 \mathrm{H}, 7^{\prime \prime}-, 8^{\prime \prime}-, 12^{\prime \prime}-, 13^{\prime \prime}\right.$-, $\left.17^{\prime \prime}-18^{\prime \prime}-\mathrm{H}\right), 8.91$ (s, $\left.1 \mathrm{H}, 3^{\prime \prime}-\mathrm{H}\right), 9.46$ (s, 1 H, CHO), 12.56 (s, 2H, $\mathrm{NH}$ ). $-\mathrm{C}_{60} \mathrm{H}_{48} \mathrm{~N}_{4} \mathrm{O}$ (841.1): ber. C 85.68, H 5.75, N 6.66; gef. C 85.77 , H 5.90, N 6.37.

Kopplung der TPP-Polyenale 8 und 9 mit 7: In einen Zweihalskolben mit Septum und Rückflußkühler mit Blasenzähler und Sicapent-Trockenrohr spritzt man unter $\mathrm{N}_{2}$ bei Raumtemp. unter Rühren zu 7 in Diethylether eine Lösung von BuLi in Hexan, gibt nach $45 \mathrm{~min}$ zur Zerstörung von überschüssigem BuLi $1 \mathrm{ml}$ Dichlormethan zu und tropft anschließend 8 bzw. 9 in Dichlormethan zu. Nach Beendigung der Reaktion (dünnschichtchromatographisch ermittelt) engt man ein und chromatographiert den Rückstand an Kieselgel. Das Produkt wird umkristallisiert und i. Hochvak. bei $50-60^{\circ} \mathrm{C}$ über Paraffin und Sicapent getrocknet.

a) 23-(9-Anthryl)-3-[11-(21,23-dihydro-5,10,15,20-tetraphenylporphyrin-2-yl)-4,9-dimethylundeca-2,4,6,8,10-pentaenyliden J-5 $\alpha$ -

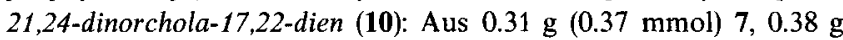
$(0.49 \mathrm{mmol}) 8$ und $0.23 \mathrm{ml}(0.37 \mathrm{mmol})$ einer $1.6 \mathrm{M}$ Lösung von BuLi in Hexan nach 20stdg. Rühren und Chromatographie mit
Dichlormethan, Chromatographie der Hauptfraktion mit Dichlormethan/Hexan (10:9) und Umkristallisation aus Dichlormethan/ Hexan; Ausb. $64 \mathrm{mg}(14 \%)$, Schmp. $231-233^{\circ} \mathrm{C}$ (Isomerengemisch). Das all-(E)-Isomer [all-(E)-Anteil $97.7 \%$ ] wird durch präparative HPLC (Hexan/Dichlormethan-Gradient, 0.4\% Dichlormethan/ $\min$, Fluß $10 \mathrm{ml} / \mathrm{min}$, Detektion bei $\lambda=550 \mathrm{~nm}$ ) von den Isomeren abgetrennt und angereichert. $-{ }^{1} \mathrm{H}-\mathrm{NMR}\left(\mathrm{CDCl}_{3}\right): \delta=0.61-2.77$ (m, Steroid-H, 4-, 9- $\mathrm{CH}_{3}$ ), darin 0.91 und 0.96 (je s, $6 \mathrm{H}, \mathrm{CH}_{3}-18$, -19), $5.90-5.94(\mathrm{~m}, 1 \mathrm{H}, 1-\mathrm{H}), 6.16\left(\mathrm{~d}, J_{20,22}=10.9 \mathrm{~Hz}, 1 \mathrm{H}, 20-\mathrm{H}\right)$, $6.24\left(\mathrm{~d}, J_{5,6}=8.9 \mathrm{~Hz}, 1 \mathrm{H}, 5-\mathrm{H}\right), 6.28\left(\mathrm{~d}, J_{3,2}=14.6 \mathrm{~Hz}, 1 \mathrm{H}, 3-\mathrm{H}\right)$, $6.42\left(\mathrm{~d}, J_{8,7}=9.9 \mathrm{~Hz}, 1 \mathrm{H}, 8-\mathrm{H}\right), 6.48\left(\mathrm{~d}, J_{10,11}=15.7 \mathrm{~Hz}, 1 \mathrm{H}, 10-\mathrm{H}\right)$, $6.54-6.69(\mathrm{~m}, 3 \mathrm{H}, 2-, 6-, 7-\mathrm{H}), 6.72\left(\mathrm{dd}, J_{22,23}=15.8 \mathrm{~Hz}, 1 \mathrm{H}, 22-\right.$ H), 7.13 (d, $1 \mathrm{H}, 11-\mathrm{H}), 7.23$ (d, $1 \mathrm{H}, 23-\mathrm{H}), 7.43-7.47$ (m, 4H, 2'-, $\left.3^{\prime}-, 6^{\prime}-, 7^{\prime}-\mathrm{H}\right), 7.71-7.81\left(\mathrm{~m}, 12 \mathrm{H}, m-, p-\mathrm{H}_{\text {Phenyl }}\right), 7.80-7.97(\mathrm{~m}, 2 \mathrm{H}$, $\left.4^{\prime}-, 5^{\prime}-\mathrm{H}\right), 8.11-8.14\left(\mathrm{~m}, 2 \mathrm{H}, 20^{\prime \prime}-\mathrm{o}-\mathrm{H}_{\text {Phenyl }}\right), 8.18-8.24(\mathrm{~m}, 6 \mathrm{H}$, $\left.5^{\prime \prime}-, 10^{\prime \prime}-, 15^{\prime \prime}-0-\mathrm{H}_{\text {Phenyl }}\right), 8.34-8.37\left(\mathrm{~m}, 3 \mathrm{H}, 1^{\prime}-, 8^{\prime}-, 10^{\prime}-\mathrm{H}\right), 8.65(\mathrm{~d}$, $\left.J_{18^{\prime \prime}, 17^{\prime \prime}}=4.8 \mathrm{~Hz}, 1 \mathrm{H}, 18^{\prime \prime}-\mathrm{H}\right), 8.73\left(\mathrm{~d}, 1 \mathrm{H}, 17^{\prime \prime}-\mathrm{H}\right), 8.75-8.81(\mathrm{~m}, 4 \mathrm{H}$, $\left.7^{\prime \prime}-, 8^{\prime \prime}-, 12^{\prime \prime}-, 13^{\prime \prime}-\mathrm{H}\right), 8.90$ (s, 1H, 3"-H), 12.56 (s, 2H, NH). $\mathrm{C}_{93} \mathrm{H}_{84} \mathrm{~N}_{4}$ (1257.7): ber. C 88.81, H 6.73, N 4.45; gef. C 88.89, H 6.69, $\mathrm{N} 4.33$.

b) 23-(9-Anthryl)-3-[15-(21,23-dihydro-5,10,15,20-tetraphenylporphyrin-2-yl)-4,8,13-trimethylpentadeca-2,4,6,8,10,12,14-heptaenyliden ]-5 $\alpha-21,24-$ dinorchola-17,22-dien (11): Aus $84 \mathrm{mg}(0.10$ $\mathrm{mmol}) 9,0.25 \mathrm{~g}(0.3 \mathrm{mmol}) 7,0.24 \mathrm{ml}(0.38 \mathrm{mmol})$ einer $1.6 \mathrm{M}$ Lösung von BuLi in Hexan nach 15stdg. Rühren, Chromatographie mit Hexan/Dichlormethan (54:46) und Umkristallisation aus Hexan/ Dichlormethan; Ausb. $57 \mathrm{mg}(43 \%)$, Schmp. $>230^{\circ} \mathrm{C}$. Das all $-(E)-$ Isomer wird durch MPLC mit Hexan/Dichlormethan (75:25) (Detektion bei $\lambda=257$ und $600 \mathrm{~nm})$ angereichert. $-{ }^{1} \mathrm{H}-\mathrm{NMR}\left(\mathrm{CDCl}_{3}\right)$ : $\delta=0.61-2.51 \quad\left(\mathrm{~m}\right.$, Steroid-H, 4-, 8-, $\left.13-\mathrm{CH}_{3}, \mathrm{CH}_{3}-18,-19\right)$, 5.87-5.92 (m, 1 H, 1-H), 6.13-6.77 (m, 12H, 2-, 3-, 5-, 6-, 7-, 9-, 10-, 11-, 12-, 14-, 20-, 22-H), 7.13 (d, $\left.J_{15,14}=15.5 \mathrm{~Hz}, 1 \mathrm{H}, 15-\mathrm{H}\right), 7.22$ $\left(\mathrm{d}, J_{23,22}=17 \mathrm{~Hz}, 1 \mathrm{H}, 23-\mathrm{H}\right), 7.43-7.47\left(\mathrm{~m}, 4 \mathrm{H}, 2^{\prime}-, 3^{\prime}-, 6^{\prime}-, 7^{\prime}-\mathrm{H}\right)$, $7.71-7.79\left(\mathrm{~m}, 12 \mathrm{H}, m-, p-\mathrm{H}_{\text {Phenyl }}\right), 7.96-8.00\left(\mathrm{~m}, 2 \mathrm{H}, 4^{\prime}-, 5^{\prime}-\mathrm{H}\right)$, $8.11-8.14\left(\mathrm{~m}, 2 \mathrm{H}, 20^{\prime \prime}-o-\mathrm{H}_{\text {Phenyl }}\right), 8.18-8.24\left(\mathrm{~m}, 6 \mathrm{H}, 5^{\prime \prime}-, 10^{\prime \prime}-, 15^{\prime \prime}-\right.$ $\left.o-\mathrm{H}_{\text {Phenyl }}\right), 8.34-8.37\left(\mathrm{~m}, 3 \mathrm{H}, 1^{\prime}-, 8^{\prime}-, 10^{\prime}-\mathrm{H}\right), 8.65\left(\mathrm{~d}, J_{18^{\circ}, 17^{\circ}}=4.8\right.$ $\left.\mathrm{Hz}, 1 \mathrm{H}, 18^{\prime \prime}-\mathrm{H}\right), 8.72-8.81\left(\mathrm{~m}, 5 \mathrm{H}, 7^{\prime \prime}-, 8^{\prime \prime}-, 12^{\prime \prime}-, 13^{\prime \prime}-, 17^{\prime \prime}-\mathrm{H}\right), 8.90$ (s, $\left.1 \mathrm{H}, 3^{\prime \prime}-\mathrm{H}\right), 12.56$ (s, $\left.2 \mathrm{H}, \mathrm{NH}\right) .-\mathrm{C}_{98} \mathrm{H}_{90} \mathrm{~N}_{4}(1323.8)$ : ber. C 88.92 , H 6.85, N 4.23; gef. C 88.53, H 6.95, N 4.09 .

3-(9,10-Dihydro-9-anthryl)-3-hydroxy-5a-androstan-17-on (14): Zu $9.0 \mathrm{~g}(49.9 \mathrm{mmol}) 12$ in $250 \mathrm{ml}$ Diethylether tropft man langsam unter Ar bei $-10^{\circ} \mathrm{C} 31.2 \mathrm{ml}$ (49.9 mmol) einer $1.6 \mathrm{M}$ Lösung von BuLi in Hexan, läßt auf Raumtemp. erwärmen, läßt noch $1 \mathrm{~h}$ rüh-

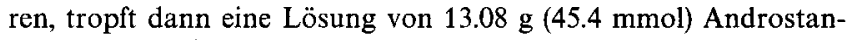
3,17-dion (13) ${ }^{[21]}$ in 11 Diethylether zu und rührt $2 \mathrm{~h}$ nach. Man gibt $5.0 \mathrm{~g}$ Trockeneis zu, rührt $5 \mathrm{~min}$, gibt dann $400 \mathrm{ml} 5$ proz. Ammoniumchlorid-Lösung zu, trennt die Phasen und filtriert den sich an der Phasengrenze angesammelten Feststoff ab. Die organische Phase wird mit Wasser neutral gewaschen, mit Natriumsulfat getrocknet und eingeengt. Der Rückstand wird an Kieselgel mit Dichlormethan/Ethylacetat (9:1) chromatographiert; Ausb. $5.83 \mathrm{~g}$ $(27 \%) .-{ }^{1} \mathrm{H}-\mathrm{NMR}\left(\mathrm{CDCl}_{3}\right): \delta=0.60-2.46(\mathrm{~m}$, Steroid-H), darin 0.60 und 0.82 (je s, $\left.6 \mathrm{H}, \mathrm{CH}_{3}-18,-19\right), 3.78\left(\mathrm{~d}, J_{10,10}=18.7 \mathrm{~Hz}, 1 \mathrm{H}\right.$, $\left.10^{\prime}-\mathrm{H}\right), 3.84$ (s, $\left.1 \mathrm{H}, 9^{\prime}-\mathrm{H}\right), 4.29$ (d, $\left.1 \mathrm{H}, 10^{\prime}-\mathrm{H}\right), 7.18-7.33(\mathrm{~m}, 8 \mathrm{H}$, Anthryl-H).

Das Produkt wird ohne weitere Reinigung weiter umgesetzt.

3-(9,10-Dihydro-9-anthryl)-5 $\alpha$-androst-2-en-17-on (15): Unter $\mathrm{N}_{2}$ erhitzt man $5.83 \mathrm{~g}(12.5 \mathrm{mmol}) 14$ und $2.0 \mathrm{~g}(11.6 \mathrm{mmol}) p$-Toluolsulfonsäure in $200 \mathrm{ml}$ Toluol zum Sieden, destilliert $100 \mathrm{ml}$ Toluol/ Wasser-Gemisch ab und gießt dann das abgekühlte Reaktionsgemisch auf Wasser. Die organische Phase wird mit verd. Natriumhydrogencarbonat-Lösung und Wasser neutral gewaschen, mit Na- 
triumsulfat getrocknet und eingeengt. Der Rückstand wird an Kieselgel mit Dichlormethan chromatographiert; Ausb. $4.62 \mathrm{~g} \mathrm{(82 \% )}$. $-{ }^{\mathrm{H}} \mathrm{H}-\mathrm{NMR}\left(\mathrm{CDCl}_{3}\right): \delta=0.56-2.45(\mathrm{~m}$, Steroid-H), darin 0.59 und 0.82 (je s, $6 \mathrm{H}, \mathrm{CH}_{3}-18,-19$ ), 3.87 und 4.08 (je d, $J_{10^{\prime}, 10^{\prime}}=18.7 \mathrm{~Hz}$, $\left.2 \mathrm{H}, 10^{\prime}-\mathrm{H}\right), 4.53\left(\mathrm{~s}, 1 \mathrm{H}, 9^{\prime}-\mathrm{H}\right), 5.29-5.32(\mathrm{~m}, 1 \mathrm{H}$, Ster-2-H), $7.12-7.31$ (m, $8 \mathrm{H}$, Anthryl-H).

Das Produkt wird ohne weitere Reinigung weiter umgesetzt.

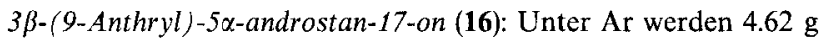
(10.3 mmol) 15 und $15.0 \mathrm{~g}(87.1 \mathrm{mmol}) p$-Toluolsulfonsäure in 100 $\mathrm{ml} \mathrm{Xylol} 3$ h unter Rückfluß erhitzt. Das abgekühite Gemisch wird auf Wasser gegossen, die organische Phase mit Natriumhydrogencarbonat-Lösung und Wasser neutral gewaschen, mit Natriumsulfat getrocknet und eingeengt. Der Rückstand wird an Kieselgel mit Dichlormethan chromatographiert; Ausb. $2.86 \mathrm{~g}$ eines Gemisches aus 15 und 16, das durch MPLC mit Hexan/Ethylacetat (95:5) (Detektion bei 257 und $370 \mathrm{~nm}$ ) getrennt wird. 1. Fraktion: Ausb. $741 \mathrm{mg} \mathrm{15}$. - 2. Fraktion: Ausb. $1.53 \mathrm{~g} \mathrm{(33 \% )} \mathrm{16,} \mathrm{das} \mathrm{aus} \mathrm{Dichlor-}$ methan mit Methanol umkristallisiert und i.Hochvak. bei $70^{\circ} \mathrm{C}$

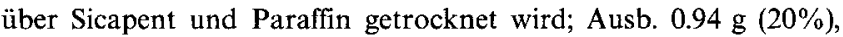
Schmp. $273^{\circ} \mathrm{C}$. - ${ }^{1} \mathrm{H}-\mathrm{NMR}\left(\mathrm{CDCl}_{3}\right): \delta=0.87-2.74$ (m, Steroid$\mathrm{H}$ ), darin 0.92 and 1.16 (je s, $\left.6 \mathrm{H}, \mathrm{CH}_{3}-18,-19\right), 4.12-4.22(\mathrm{~m}, 1 \mathrm{H}$, Ster-3-H), 7.40-7.48 (m, 4H, 2'-, 3'-, 6'-, 7'-H), 7.79-8.01 (m, 2H, $\left.4^{\prime}-, 5^{\prime}-\mathrm{H}\right), 8.32\left(\mathrm{~s}, 1 \mathrm{H}, 10^{\prime}-\mathrm{H}\right), 8.32-8.62\left(\mathrm{~m}, 2 \mathrm{H}, 1^{\prime}-, 8^{\prime}-\mathrm{H}\right)$. $\mathrm{C}_{33} \mathrm{H}_{38} \mathrm{O}$ (450.7): ber. C 87.95, H 8.50; gef. C 87.87, H 8.62.

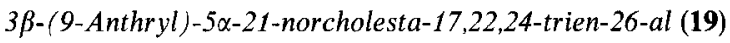

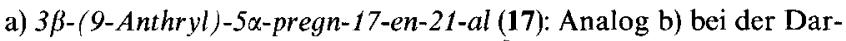
stellung von 3, jedoch Aufnehmen in Dichlormethan und Neutralwaschen mit Wasser, Einengen, Aufnehmen in Benzol; Extrahieren mit Benzol und Chromatographieren an Kieselgel mit Dichlormethan sowie MPLC mit Hexan/Dichlormethan (94:6) (Detektion bei 259 und $300 \mathrm{~nm})$; aus $0.57 \mathrm{~g}(1.27 \mathrm{mmol}) 16$ und $1.65 \mathrm{~g} \mathrm{(6.32}$ mmol) 2 in $40 \mathrm{ml} \mathrm{THF}, 3.95 \mathrm{ml}(6.32 \mathrm{mmol})$ einer $1.6 \mathrm{M}$ Lösung von BuLi in Hexan und $60 \mathrm{ml} 1$ proz. Oxalsäure; Ausb. $0.42 \mathrm{~g}$ $(69 \%)$, Schmp. $232^{\circ} \mathrm{C}$. $-{ }^{\mathrm{t}} \mathrm{H}-\mathrm{NMR}\left(\mathrm{CDCl}_{3}\right): \delta=0.82-2.98(\mathrm{~m}$, Steroid-H), darin 0.89 und 1.16 (je s, $\left.6 \mathrm{H}, \mathrm{CH}_{3}-18,-19\right), 4.11-4.22$ (m, $1 \mathrm{H}$, Ster-3-H), $5.76\left(\mathrm{td}, J_{20, \mathrm{CHO}}=8.0,{ }^{4} J_{20,16}=2.2 \mathrm{~Hz}, 1 \mathrm{H}, 20-\mathrm{H}\right)$, $7.39-7.50\left(\mathrm{~m}, 4 \mathrm{H}, 2^{\prime}-, 3^{\prime}-, 6^{\prime}-, 7^{\prime}-\mathrm{H}\right), 7.96-8.00\left(\mathrm{~m}, 2 \mathrm{H}, 4^{\prime}-, 5^{\prime}-\mathrm{H}\right)$, 8.31 (s, $\left.1 \mathrm{H}, 10^{\prime}-\mathrm{H}\right), 8.31-8.41\left(\mathrm{~m}, 2 \mathrm{H}, 1^{\prime}-, 8^{\prime}-\mathrm{H}\right), 9.88$ (d, $\left.1 \mathrm{H}, \mathrm{CHO}\right)$. $-\mathrm{C}_{35} \mathrm{H}_{40} \mathrm{O}$ (476.7): ber. C 88.19, H 8.46; gef. C 88.16, H 8.46.

b) Bei Raumtemp. tropft man unter Ar zu $9.44 \mathrm{ml}(9.44 \mathrm{mmol})$ einer $1 \mathrm{M}$ Lösung von Natriummethanolat in Methanol und $0.90 \mathrm{~g}$ (1.89 mmol) 17 in $70 \mathrm{ml}$ Dichlormethan innerhalb $0.5 \mathrm{~h} 9.16 \mathrm{ml}$ (9.44 mmol) einer $1.03 \mathrm{M}$ Lösung von 18 in Methanol, rührt $15 \mathrm{~h}$ nach und gießt das Reaktionsgemisch auf Wasser. Die organische Phase wird mit verd. Ammoniumchlorid-Lösung und Wasser gewaschen, mit Natriumsulfat getrocknet und eingeengt. Der Rückstand wird in $60 \mathrm{ml}$ Aceton aufgenommen und mit $2 \mathrm{ml} 0.1 \mathrm{~N} \mathrm{HCl}$ versetzt, wobei das Produkt ausfällt. Nach vollständiger Fällung durch Zugabe von $50 \mathrm{ml}$ Wasser und Entfernen von Aceton i. Vak. wird der Niederschlag abfiltriert, getrocknet, in Dichlormethan aufgenommen und an Kieselgel mit Dichlormethan chromatographiert; Ausb. $0.91 \mathrm{~g}$ Isomerengemisch (mit HPLC bestimmt), das durch MPLC mit Hexan/Ethylacetat (94:6) (Detektion bei 259 und

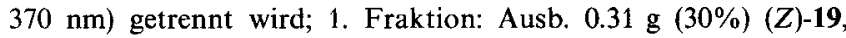
Schmp. $243-245^{\circ} \mathrm{C}$. -2 . Fraktion: $0.39 \mathrm{~g}(38 \%)$ all-(E)-19, Schmp. $226-230^{\circ} \mathrm{C}$ - ${ }^{1} \mathrm{H}-\mathrm{NMR}\left(\mathrm{CDCl}_{3}\right): \delta=0.88-2.67(\mathrm{~m}$, Steroid-H), darin 0.88, 1.19 und 1.90 (je s, $\left.9 \mathrm{H}, \mathrm{CH}_{3}-18,-19,-27\right), 4.21$ (tt, $1 \mathrm{H}$, Ster-3-H), $5.96\left(\mathrm{~d}, J_{20,22}=11.3 \mathrm{~Hz}, 1 \mathrm{H}, 20-\mathrm{H}\right), 6.57$ (dd, $J_{23,22}=14.5$, $\left.J_{23,24}=11.3 \mathrm{~Hz}, 1 \mathrm{H}, 23-\mathrm{H}\right), 6.79(\mathrm{dd}, 1 \mathrm{H}, 22-\mathrm{H}), 6.93(\mathrm{~d}, 1 \mathrm{H}, 24-\mathrm{H})$, $7.43-7.51\left(\mathrm{~m}, 4 \mathrm{H}, 2^{\prime}-, 3^{\prime}-, 6^{\prime}-, 7^{\prime}-\mathrm{H}\right), 8.00-8.03\left(\mathrm{~m}, 2 \mathrm{H}, 4^{\prime}-, 5^{\prime}-\mathrm{H}\right)$, $8.34\left(\mathrm{~s}, 1 \mathrm{H}, 10^{\prime}-\mathrm{H}\right), 8.34-8.65\left(\mathrm{~m}, 2 \mathrm{H}, 1^{\prime}-, 8^{\prime}-\mathrm{H}\right), 9.45(\mathrm{~s}, 1 \mathrm{H}, \mathrm{CHO})$ $-\mathrm{C}_{40} \mathrm{H}_{46} \mathrm{O}(542.8)$ : ber. C 88.51, $\mathrm{H} 8.54$; gef. C 88.57, H $8.61(Z)$ 19: C 88.64, H 8.68 all-(E)-19.
[3-(21,23-Dihydro-5,10,15,20-tetraphenylporphyrin-2-yl)-2-propenyl ltriphenylphosphoniumbromid (20)

a) 3-(21,23-Dihydro-5,10,15,20-tetraphenylporphyrin-2-yl)acryl-

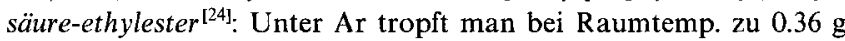
(15.0 mmol) Natriumhydrid in $70 \mathrm{ml}$ THF $3.59 \mathrm{~g}(16.0 \mathrm{mmol})$ [(Ethoxycarbonyl)methyl]phosphonsäure-diethylester ${ }^{[23]}$ in $100 \mathrm{ml}$ THF. Nach Beendigung der $\mathbf{H}_{2}$-Entwicklung wird die Lösung von $1.0 \mathrm{~g}$ (1.56 mmol) 2-Formyl-21,23-dihydro-5,10,15,20-tetraphenylporphyrin $^{[22 a]}$ in $120 \mathrm{ml}$ Dichlormethan zugegeben und $16 \mathrm{~h}$ gerührt. Das Reaktionsgemisch wird eingeengt, in Dichlormethan aufgenommen, die Lösung viermal mit Wasser gewaschen, mit Natriumsulfat getrocknet und eingeengt. Der Rückstand wird an Kieselgel mit Dichlormethan chromatographiert und das Produkt aus Dichlormethan/Hexan umkristallisiert; Ausb. $0.95 \mathrm{~g}(85 \%)$, Schmp. $>270^{\circ} \mathrm{C} .-{ }^{1} \mathrm{H}-\mathrm{NMR}\left(\mathrm{CDCl}_{3}\right): \delta=1.36(\mathrm{t}, J=7.1 \mathrm{~Hz}, 3 \mathrm{H}$, $\left.\mathrm{CH}_{3}\right), 4.23$ (q, 2H, $\left.\mathrm{CH}_{2}\right), 6.57$ (d, $\left.J_{2,3}=15.6 \mathrm{~Hz}, 1 \mathrm{H}, 2-\mathrm{H}\right), 7.42(\mathrm{dd}$, $J=0.75 \mathrm{~Hz}, 1 \mathrm{H}, 3-\mathrm{H}), 7.71-7.84\left(\mathrm{~m}, 12 \mathrm{H}, m-, p-\mathrm{H}_{\mathrm{Phenyl}}\right), 8.11-8.21$ $\left(\mathrm{m}, 8 \mathrm{H}\right.$, o- $\left.\mathrm{H}_{\text {Phenyl }}\right), 8.76-8.83\left(\mathrm{~m}, 6 \mathrm{H}, 7^{\prime \prime}-, 8^{\prime \prime}-, 12^{\prime \prime}-, 13^{\prime \prime}-, 7^{\prime \prime}-\right.$, $\left.18^{\prime \prime}-\mathrm{H}\right), 8.97$ (s, 1 H, 3"-H), 12.61 (s, 2H, NH).

b) 21,23-Dihydro-2-(3-hydroxy-1-propenyl)-5,10,15,20-tetraphenylporphyrin: Unter Ar tropft man bei $0^{\circ} \mathrm{C} \mathrm{zu} 0.95 \mathrm{~g}(1.33 \mathrm{mmol})$ 3-(21,23-Dihydro-5,10,15,20-tetraphenylporphyrin-2-yl)acrylsäureethylester in $300 \mathrm{ml}$ THF $6.66 \mathrm{ml}(6.66 \mathrm{mmol})$ einer $1 \mathrm{~N}$ Lösung von Diisobutylaluminiumhydrid (DIBAL) in THF. Nach vollständigem Umsatz (DC-Kontrolle) werden je $10 \mathrm{ml}$ Methanol und Wasser zugegeben. Das Reaktionsgemisch wird i.Vak. eingeengt und in Dichlormethan aufgenommen. Nach Abfiltrieren der unlöslichen Bestandteile wird an Kieselgel mit Dichlormethan/Ethylacetat (10:1) chromatographiert. Die Hauptfraktion wird aus Dichlormethan/Hexan umkristallisiert und bei $60^{\circ} \mathrm{C}$ i. Hochvak. über Paraffin und Sicapent getrocknet; Ausb. $0.68 \mathrm{~g}(76 \%)$, Schmp. $>300^{\circ} \mathrm{C}$. $-{ }^{1} \mathrm{H}$-NMR $\left(\mathrm{CDCl}_{3}\right): \delta=1.06\left(\mathrm{t}, J_{\mathrm{CH}_{2}, \mathrm{OH}}=6.3 \mathrm{~Hz}, 1 \mathrm{H}, \mathrm{OH}\right), 4.12$ $\left(\mathrm{dd}, J_{\mathrm{CH}_{2}, 2}=5.4 \mathrm{~Hz}, 2 \mathrm{H}, \mathrm{CH}_{2}\right), 6.28\left(\mathrm{dq}, J_{1,2}=15.6, J_{1, \mathrm{CH}_{2}}=1.2 \mathrm{~Hz}\right.$, $1 \mathrm{H}, 1-\mathrm{H}), 6.48(\mathrm{td}, 1 \mathrm{H}, 2-\mathrm{H}), 7.69-7.82\left(\mathrm{~m}, 12 \mathrm{H}, m-, p-\mathrm{H}_{\text {Phenyl }}\right)$, $8.08-8.11\left(\mathrm{~m}, 2 \mathrm{H}, 20^{\prime \prime}-o-\mathrm{H}_{\text {Phenyl }}\right), 8.18-8.22\left(\mathrm{~m}, 6 \mathrm{H}, 5^{\prime \prime}-, 10^{\prime \prime}-, 15^{\prime \prime}\right.$ $\left.o-\mathrm{H}_{\text {Phenyl }}\right), 8.73\left(\mathrm{~d}, J_{18^{\prime \prime}, 17^{\prime \prime}}=4.8 \mathrm{~Hz}, 1 \mathrm{H}, 18^{\prime \prime}-\mathrm{H}\right), 8.77-8.83(\mathrm{~m}, 6 \mathrm{H}$, $\left.3^{\prime \prime}-, 7^{\prime \prime}-, 8^{\prime \prime}-, 12^{\prime \prime}-, 13^{\prime \prime}-, 17^{\prime \prime}-\mathrm{H}\right), 12.70$ (s, $\left.2 \mathrm{H}, \mathrm{NH}\right) .-\mathrm{C}_{47} \mathrm{H}_{34} \mathrm{~N}_{4} \mathrm{O}$ (670.8): ber. C 84.15, H 5.11, N 8.35; gef. C 83.91, H 5.05, N 8.21.

c) 2-(3-Brom-1-propenyl)-21,23-dihydro-5,10,15,20-tetraphenylporphyrin: Unter Ar gibt man zu $0.71 \mathrm{~g}$ (1.06 mmol) 21,23-Dihydro2-(3-hydroxy-1-propenyl)-5,10,15,20-tetraphenylporphyrin in 150 $\mathrm{ml}$ 1,2-Dichlorethan $50 \mathrm{ml}$ Dimethylformamid (DMF) und spritzt bei $0^{\circ} \mathrm{C}$ innerhalb $5 \mathrm{~min} 0.88 \mathrm{ml}(9.36 \mathrm{mmol})$ Phosphortribromid zu. Man läßt $1 \mathrm{~h}$ rühren und wäscht dann jeweils zweimal mit eiskaltem Wasser, verd. Natriumhydrogencarbonat-Lösung und Wasser, trocknet mit Natriumsulfat, engt i.Vak. ein und setzt das Produkt direkt ohne Reinigung weiter um. - ${ }^{1} \mathrm{H}-\mathrm{NMR}\left(\mathrm{CDCl}_{3}\right)$ : $\delta=3.90\left(\mathrm{~d}, J_{\mathrm{CH}_{2,2}}=7.7 \mathrm{~Hz}, 2 \mathrm{H}, \mathrm{CH}_{2}\right), 6.16\left(\mathrm{~d}, J_{1,2}=15.2 \mathrm{~Hz}, 1 \mathrm{H}\right.$, $1-\mathrm{H}), 6.50(\mathrm{td}, 1 \mathrm{H}, 2-\mathrm{H}), 7.62-7.77\left(\mathrm{~m}, 12 \mathrm{H}, m-p-\mathrm{H}_{\text {Pheny } 1}\right)$, $7.96-8.06\left(\mathrm{~m}, 2 \mathrm{H}, 20^{\prime \prime}-o-\mathrm{H}_{\text {Phenyl }}\right), 8.10-8.21\left(\mathrm{~m}, 6 \mathrm{H}, 5^{\prime \prime}\right.$-, $10^{\prime \prime}$-, $15^{\prime \prime}-$ $\left.o-\mathrm{H}_{\text {Phenyl) }}\right), 8.64-8.73$ (m, 7H, 3"-, 7"-, 8"-, 12"-, 13"-, 17"-, 18"-H), 12.67 (s, $2 \mathrm{H}, \mathrm{NH})$.

d) Unter Ar gibt man zum Rohprodukt in $90 \mathrm{ml}$ Toluol $0.83 \mathrm{~g}$ (3.16 mmol) Triphenylphosphan und erhitzt $6 \mathrm{~h}$ unter Rückfluß, wobei ein Niederschlag ausfällt. Nach Abkühlen gibt man $100 \mathrm{ml}$ Diethylether zu, filtriert den Niederschlag ab und kristallisiert aus Dichlormethan/Ethylacetat sowie Aceton/Hexan um; Ausb. $297 \mathrm{mg}$ 20 [28\% bezogen auf 21,23-Dihydro-2-(3-hydroxy-1-propenyl)$5,10,15,20$-tetraphenylporphyrin], Schmp. $>230^{\circ} \mathrm{C}$. $-{ }^{1} \mathrm{H}-\mathrm{NMR}$ $\left(\mathrm{CDCl}_{3}\right): \delta=4.55\left(\mathrm{dd}, J_{\mathrm{CH}_{2,2}}=6.8, J_{\mathrm{CH}_{2}, \mathrm{P}}=14.8 \mathrm{~Hz}, 2 \mathrm{H}, \mathrm{CH}_{2} \mathrm{P}\right)$, $5.91-6.12(\mathrm{~m}, 2 \mathrm{H}, 2-, 3-\mathrm{H}), 7.57-7.84\left(\mathrm{~m}, 27 \mathrm{H}, m-, p-\mathrm{H}_{\mathrm{Phenyl}}\right.$, $\left.\mathrm{H}_{\text {Pheny }}\right), 7.94$ (d, $J=6.9 \mathrm{~Hz}, 2 \mathrm{H}, 20$ " $\left.-o-\mathrm{H}_{\text {Phenyl }}\right), 8.14-8.20(\mathrm{~m}, 6 \mathrm{H}$, 
5"-, 10"-, 15"-o-H $\left.\mathrm{H}_{\text {Pheny }}\right), 8.54$ (s, $\left.1 \mathrm{H}, 3^{\prime \prime}-\mathrm{H}\right), 8.70\left(\mathrm{~d}, J_{18^{\prime \prime}, 17^{\circ}}=4.9 \mathrm{~Hz}\right.$, $\left.1 \mathrm{H}, 18^{\prime \prime}-\mathrm{H}\right), 8.76-8.80$ (m, 5H, 7"-, 8"-, 12"-, 13"-, 17"-H), 12.73 (s, $2 \mathrm{H}, \mathrm{NH}$ ). $-\mathrm{C}_{65} \mathrm{H}_{48} \mathrm{BrN}_{4} \mathrm{P}$ (996.0): ber. C 78.38, H 4.86, Br 8.02, N 5.63; gef. C 78.21, H 4.78, Br 8.21, N 5.35.

33-(9-Anthryl)-17-[9-(21,23-dihydro-5,10,15,20-tetraphenylporphyrin-2-yl)-5-methylnona-2,4,6,8-tetraenyliden $]-5 \alpha$-androstan (21): Unter Ar spritzt man $20 \mathrm{ml}$ Methanol und $0.28 \mathrm{ml}(0.28 \mathrm{mmol})$ einer $1 \mathrm{~N}$ Lösung von Natriummethanolat in Methanol zu der Lösung von $0.14 \mathrm{~g}(0.26 \mathrm{mmol}) 19$ in $20 \mathrm{ml}$ Dichlormethan, tropft dann innerhalb $10 \mathrm{~min}$ eine Lösung von $0.28 \mathrm{~g}(0.28 \mathrm{mmol}) 20$ in $20 \mathrm{ml}$ Dichlormethan zu und rührt $15 \mathrm{~h}$ nach. Das Reaktionsgemisch wird dreimal mit Wasser gewaschen, mit Natriumsulfat getrocknet und eingeengt. Der Rückstand wird an Kieselgel mit Dichlormethan chromatographiert und all-(E)-21 durch MPLC mit Hexan/Dichlormethan (75:25) gereinigt und angereichert; Ausb. $0.10 \mathrm{~g}(32 \%)$, Schmp. $>252{ }^{\circ} \mathrm{C}$. $-{ }^{1} \mathrm{H}-\mathrm{NMR}\left(\mathrm{CDCl}_{3}\right): \delta=0.60-2.90$ (m, Steroid$\mathrm{H})$, darin $0.86,1.17$ und $1.96\left(\mathrm{je} \mathrm{s}, 9 \mathrm{H}, \mathrm{CH}_{3}-18,-19,-25\right), 4.18-4.24$ $\left(\mathrm{m}, 1 \mathrm{H}\right.$, Ster-3-H), $5.90\left(\mathrm{~d}, J_{20,22}=10.8 \mathrm{~Hz}, 1 \mathrm{H}, 20-\mathrm{H}\right), 6.10(\mathrm{dd}$, $\left.J_{27,26}=15.2, J_{27,28}=10.8 \mathrm{~Hz}, 1 \mathrm{H}, 27-\mathrm{H}\right), 6.15(\mathrm{~d}, 1 \mathrm{H}, 26-\mathrm{H}), 6.25(\mathrm{~d}$, $\left.J_{24,23}=10.5 \mathrm{~Hz}, 1 \mathrm{H}, 24-\mathrm{H}\right), 6.42\left(\mathrm{dd}, J_{23,22}=14.6 \mathrm{~Hz}, 1 \mathrm{H}, 23-\mathrm{H}\right)$, $6.49\left(\mathrm{~d}, J_{29.28}=15.0 \mathrm{~Hz}, 1 \mathrm{H}, 29-\mathrm{H}\right), 6.54(\mathrm{dd}, 1 \mathrm{H}, 22-\mathrm{H}), 7.09(\mathrm{dd}$, $1 \mathrm{H}, 28-\mathrm{H}), 7.40-7.54\left(\mathrm{~m}, 4 \mathrm{H}, 2^{\prime}-, 3^{\prime}-, 6^{\prime}-, 7^{\prime}-\mathrm{H}\right), 7.69-7.85(\mathrm{~m}, 12 \mathrm{H}$, $m-, p-\mathrm{H}_{\text {Phenyl) }}$ ) $7.97-8.01$ (m, 2H, 4'-, 5'-H), $8.09-8.13$ (m, 2H, 20" $\left.o-\mathrm{H}_{\text {Phenyl }}\right), 8.18-8.24\left(\mathrm{~m}, 6 \mathrm{H}, 5^{\prime \prime}-, 0^{\prime \prime}-, 15^{\prime \prime}-o-\mathrm{H}_{\text {Phenyl }}\right), 8.32(\mathrm{~s}, 1 \mathrm{H}$, $10^{\prime}-\mathrm{H}$ ), 8.42 und 8.63 (je s, $\left.2 \mathrm{H}, 1^{\prime}-, 8^{\prime}-\mathrm{H}\right), 8.72-8.82\left(\mathrm{~m}, 6 \mathrm{H}, 7^{\prime \prime}-\right.$,

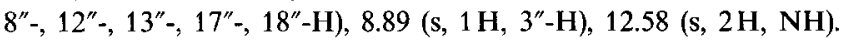
$-\mathrm{C}_{87} \mathrm{H}_{78} \mathrm{~N}_{4}$ (1179.6): ber. C 88.59, H 6.66, N 4.75; gef. C 88.37, H $6.67, \mathrm{~N} 4.63$.

3B-(9-Anthryl)-17-[5-(21,23-dihydro-5,10,15,20-tetraphenylporphyrin-2-yl) penta-2,4,6-trienyliden $/-5 \alpha$-androstan (22): Analog 21 aus $0.12 \mathrm{~g}(0.25 \mathrm{mmol}) \mathbf{1 7}, 0.50 \mathrm{~g}(0.50 \mathrm{mmol}) 20$ und $0.50 \mathrm{ml}(0.50$ mmol) einer $1 \mathrm{~N}$ Lösung von Natriummethanolat in Methanol; Umkristallisieren aus Benzol/Hexan, Ausb. $53 \mathrm{mg}(19 \%)$, Schmp. $>232^{\circ} \mathrm{C}$. $-{ }^{1} \mathrm{H}-\mathrm{NMR}\left(\mathrm{CDCl}_{3}\right): \delta=0.82-2.72(\mathrm{~m}$, Steroid-H), darin 0.89 und 1.20 (je s, $\left.6 \mathrm{H}, \mathrm{CH}_{3}-18,-19\right), 4.22$ (tt, $1 \mathrm{H}$, Ster-3-H), 5.87 $\left(\mathrm{d}, J_{20,22}=11.1 \mathrm{~Hz}, 1 \mathrm{H}, 20-\mathrm{H}\right), 6.14\left(\mathrm{~d}, J_{25,24}=15.1 \mathrm{~Hz}, 1 \mathrm{H}, 25-\mathrm{H}\right)$, $6.53\left(\mathrm{dd}, J_{22.23}=14.8 \mathrm{~Hz}, 1 \mathrm{H}, 22-\mathrm{H}\right), 7.13\left(\mathrm{dd}, J_{24,23}=10.9 \mathrm{~Hz}, 1 \mathrm{H}\right.$, 24-H), $7.42-7.54\left(\mathrm{~m}, 4 \mathrm{H}, 2^{\prime}-, 3^{\prime}-, 6^{\prime}-, 7^{\prime}-\mathrm{H}\right), 7.72-7.91(\mathrm{~m}, 12 \mathrm{H}$, $\left.m-, p-\mathrm{H}_{\text {Phenyl }}\right), 7.99-8.03\left(\mathrm{~m}, 2 \mathrm{H}, 4^{\prime}-, 5^{\prime}-\mathrm{H}\right), 8.12-8.15\left(\mathrm{~m}, 2 \mathrm{H}, 20^{\prime \prime}-\right.$ $\left.o-\mathrm{H}_{\text {Phenyl }}\right), 8.22-8.30\left(\mathrm{~m}, 6 \mathrm{H}, 5^{\prime \prime}-, 10^{\prime \prime}-, 15^{\prime \prime}-o-\mathrm{H}_{\text {Phenyi }}\right), 8.34$ (s, $1 \mathrm{H}$, $\left.10^{\prime}-\mathrm{H}\right), 8.34-8.69\left(\mathrm{~m}, 2 \mathrm{H}, 1^{\prime}-, 8^{\prime}-\mathrm{H}\right), 8.72-8.88\left(\mathrm{~m}, 6 \mathrm{H}, 7^{\prime \prime}-, 8^{\prime \prime}-\right.$, $\left.12^{\prime \prime}-, 13^{\prime \prime}-, 17^{\prime \prime}-, 18^{\prime \prime}-\mathrm{H}\right), 8.94$ (s, $\left.1 \mathrm{H}, 3^{\prime \prime}-\mathrm{H}\right), 12.53$ (s, $\left.2 \mathrm{H}, \mathrm{NH}\right)$. $\mathrm{C}_{82} \mathrm{H}_{72} \mathrm{~N}_{4}(1113.5)$ : ber. C 88.45, H 6.52, N 5.03; gef. C 88.02, H 6.55, N 4.81 .

\section{5-(9-Anthryl)-2-methylpenta-2,4-dienal (24): Nach Lit. ${ }^{[26]}$.}

[(21,23-Dihydro-5,10,15,20-tetraphenylporphyrin-2-yl)methyl]triphenylphosphoniumbromid (25): Analog c) und d) bei der Darstellung von 20; aus $0.42 \mathrm{~g}(0.65 \mathrm{mmol}) 21,23$-Dihydro-2-(hydroxymethyl)-5,10,15,20-tetraphenylporphyrin ${ }^{\text {[22a] }}, 30 \mathrm{ml} \mathrm{DMF}, 1.41 \mathrm{~g}$ (5.20 mmol) Phosphortribromid sowie $0.51 \mathrm{~g}(1.95 \mathrm{mmol})$ Triphenylphosphan, 3stdg. Erhitzen unter Rückfluß und Umkristallisieren aus wäßrigem Methanol/Diethylether; Ausb. $0.27 \mathrm{~g} \mathrm{(39 \% ),} \mathrm{Schmp.}$ $269^{\circ} \mathrm{C}$. $-{ }^{1} \mathrm{H}-\mathrm{NMR}\left(\mathrm{CDCl}_{3}\right): \delta=1.21\left(\mathrm{t}, J=7.0 \mathrm{~Hz}, 6 \mathrm{H}, \mathrm{CH}_{3}\right), 1.80$ $\left(\mathrm{s}, 2 \mathrm{H}, \mathrm{H}_{2} \mathrm{O}\right), 3.48\left(\mathrm{q}, 4 \mathrm{H}, \mathrm{OCH}_{2}\right), 5.16\left(\mathrm{~d}, J_{\mathrm{CH}_{2}, \mathrm{P}}=14.9 \mathrm{~Hz}, 2 \mathrm{H}\right.$, $\left.\mathrm{CH}_{2} \mathrm{P}\right), 7.08-7.16\left(\mathrm{~m}, 6 \mathrm{H}, 5^{\prime \prime}-, 20^{\prime \prime}-m-, p-\mathrm{H}_{\text {Phenyl }}\right), 7.27-7.33(\mathrm{~m}, 6 \mathrm{H}$, $\left.10^{\prime \prime}-, 15^{\prime \prime}-m-, p-\mathrm{H}_{\text {Phenyl }}\right), 7.40\left(\mathrm{~d}, J=6.9 \mathrm{~Hz}, 2 \mathrm{H}, 5^{\prime \prime}-, 20^{\prime \prime}-o-\mathrm{H}_{\text {Phenyl }}\right)$, $7.54-7.91\left(\mathrm{~m}, 17 \mathrm{H}, \mathrm{H}_{\text {Phenyl, }} 5^{\prime \prime}-\right.$, 20"-o- $\left.\mathrm{H}_{\text {Phenyl }}\right), 8.17$ (dd, $J_{1}=7.7$, $\left.J_{2}=1.1 \mathrm{~Hz}, 4 \mathrm{H}, 10^{\prime \prime}-, 15^{\prime \prime}-o-\mathrm{H}_{\text {Pheny }}\right), 8.29\left(\mathrm{~d}, J=3.5 \mathrm{~Hz}, 1 \mathrm{H}, \mathrm{H}_{\text {Porph }}\right)$, $8.46\left(\mathrm{~d}, J=5.0 \mathrm{~Hz}, 1 \mathrm{H}, \mathrm{H}_{\text {Porph }}\right), 8.75-8.87\left(\mathrm{~m}, 5 \mathrm{H}, \mathrm{H}_{\text {Porph }}\right), 12.78(\mathrm{~s}$, $2 \mathrm{H}, \mathrm{NH}$ ). $-\mathrm{C}_{63} \mathrm{H}_{46} \mathrm{BrN}_{4} \mathrm{P} \cdot \mathrm{H}_{2} \mathrm{O} \cdot \mathrm{C}_{2} \mathrm{H}_{5} \mathrm{OC}_{2} \mathrm{H}_{5}$ (1062.1): ber. C 75.77, H 5.50, N 5.28, P 2.92; gef. C 75.79, H 5.27, N 5.24, P 2.68.
6-(9-Anthryl)-1-(21,23-dihydro-5,10,15,20-tetraphenylporphyrin2-yl)-3-methyl-1,3,5-hexatrien (26): Analog 21 aus $2.11 \mathrm{~g}$ (7.75 mmol) 24 in $150 \mathrm{ml}$ Dichlormethan, $1.55 \mathrm{ml}(1.55 \mathrm{mmol})$ einer $1 \mathrm{~N}$ Lösung von Natriummethanolat in Methanol und $1.50 \mathrm{~g}$ (1.55 mmol) 25 in $70 \mathrm{ml}$ Methanol; Reinigen und Anreichern von all-(E)26 durch MPLC mit Hexan/Dichlormethan (80:20) und Umkristallisieren aus Benzol/Hexan (1:1); Ausb. $0.41 \mathrm{~g}$ (30\%), Schmp. $308.5^{\circ} \mathrm{C}$. $-{ }^{1} \mathrm{H}-\mathrm{NMR}\left(\mathrm{CDCl}_{3}\right): \delta=1.68\left(\mathrm{~s}, 3 \mathrm{H}, \mathrm{CH}_{3}\right), 6.56$ (d, $\left.J_{2,1}=15.6 \mathrm{~Hz}, 1 \mathrm{H}, 2-\mathrm{H}\right), 6.70\left(\mathrm{~d}, J_{4,5}=11.3 \mathrm{~Hz}, 1 \mathrm{H}, 4-\mathrm{H}\right), 7.02(\mathrm{dd}$, $\left.J_{5,6}=15.6 \mathrm{~Hz}, 1 \mathrm{H}, 5-\mathrm{H}\right), 7.25(\mathrm{~d}, 1 \mathrm{H}, 1-\mathrm{H}), 7.35-7.44\left(\mathrm{~m}, 5 \mathrm{H}, 2^{\prime}-\right.$, $\left.3^{\prime}-, 6^{\prime}-, 7^{\prime}-, 6-\mathrm{H}\right), 7.68-7.78\left(\mathrm{~m}, 12 \mathrm{H}, m-, p-\mathrm{H}_{\text {Phenyl }}\right), 7.87-7.91(\mathrm{~m}$, $\left.2 \mathrm{H}, 4^{\prime}-, 5^{\prime}-\mathrm{H}\right), 8.09-8.27\left(\mathrm{~m}, 11 \mathrm{H}, o-\mathrm{H}_{\text {Phenyl, }} 1^{\prime}-, 8^{\prime}-, 10^{\prime}-\mathrm{H}\right), 8.66(\mathrm{~d}$, $\left.J_{18,17^{\prime \prime}}=4.8 \mathrm{~Hz}, 1 \mathrm{H}, 18^{\prime \prime}-\mathrm{H}\right), 8.74-8.83$ (m, 5H, 7"-, 8"-, 12"-, 13"-, $\left.17^{\prime \prime}-\mathrm{H}\right), 9.00$ (s, $\left.1 \mathrm{H}, 3^{\prime \prime}-\mathrm{H}\right), 12.55$ (s, $\left.2 \mathrm{H}, \mathrm{NH}\right) .-\mathrm{C}_{65} \mathrm{H}_{46} \mathrm{~N}_{4}$ (883.1): ber. C 88.41, H 5.25, N 6.34; gef. C 88.13, H 5.35, N 6.25.

${ }^{[1]}$ H. Strobel, Dissertation, Univ. Stuttgart, 1992.

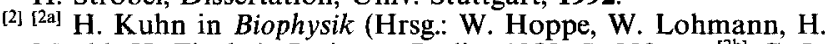
Markl, H. Ziegler), Springer, Berlin, 1982, S. 289. - ${ }^{[2 b]}$ G. L. Closs, J. R. Miller, Science 1988, 240, 440-447. - ${ }^{[2 c]}$ H. Heitele, P. Finckh, S. Weeren, F. Pöllinger, M. E. Michel-Beyerle, $J$. Phys. Chem. 1989, 93, 5173-5179. - ${ }^{[2 \mathrm{~d}]}$ J. A. Schmidt, A. R. McIntosh, A. C. Weedon, J. R. Bolton, S. J. Connolly, J. K. Hurley, M. R. Wasielewski, J. Am. Chem. Soc. 1988, 110, 1733-1740. - ${ }^{[2 \mathrm{e}]}$ F. C. De Schryver, P. Collart, J. Vandendriessche, R. Goedeweeck, A. Swinnen, M. Van der Auweraer, Acc. Chem. Res. 1987, 20,159-166. - ${ }^{127}$ R. W. Munn, Chem. Br. 1984, 20, $518-524$.

${ }^{[3]}{ }_{[3 a]}$ M. Calvin, Acc. Chem. Res. 1978, 11, 369-374. - ${ }^{[3 b]} \mathrm{H}$. Kuhn, Phys. Rev. A 1986, 34, 3409-3425. - ${ }^{[3 c]}$ A. D. Joran, B. A. Leland, P. M. Felker, A. H. Zewail, J. J. Hopfield, P. B. Dervan, Nature (London) 1987, 327, 508-511. - [3d] G McLendon, Acc. Chem. Res. 1988, 21,160-167. - ${ }^{\mid 3 e l}$ V. V. Borovkov, R. P. Evstigneeva, L. N. Strekova, E. I. Filippovich, R. F. Khairutdinov, Usp. Khim. 1989, 58, 1032-1063.

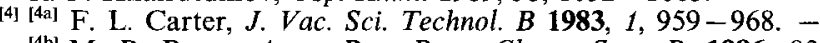
${ }^{[4 b]}$ M. R. Bryce, Annu. Rep. Prog. Chem., Sect. B, 1986, 82,

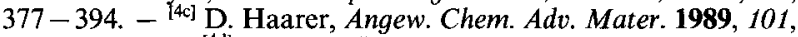
1576-1579. - ${ }^{[4 d]}$ J. J. Hopfield, J. N. Onuchic, D. N. Beratan, Science 1988, 241, 817-820. - ${ }^{[4 e]}$ J. M. Lehn, Angew. Chem. 1988, 100, 91-116; Angew. Chem. Int. Ed. Engl. 1988, 27, 89-115. - ${ }^{[4]}$ A. E. Underhill, Chem. Br. 1991, 27, 708-711. - ${ }^{[4 \mathrm{~g}]}$ D. D. C. Bradley, Chem. Br. 1991, 27, 719-723. - ${ }^{[4 h]} \mathrm{J}$. Barker, Chem. Br. 1991, 27, 728-731.

${ }^{[5]}{ }_{[\text {[a] }}$ F. Effenberger, H. Schlosser, P. Bäuerle, S. Maier, H. Port, H. C. Wolf, Angew. Chem. 1988, 100, 274-277; Angew. Chem. Int. Ed. Engl. 1988, 27, 281-284. - ${ }^{15 b]}$ S. Maier, H. Port, H. C. Wolf, F. Effenberger, H. Schlosser, Synth. Met. 1989, 29, E517-E524. - ${ }^{[\mathrm{sc}]}$ B. Heine, E. Sigmund, S. Maier, H. Port, H. C. Wolf, F. Effenberger, H. Schlosser, J. Mol. Electronics 1990 , $6,51-60$

${ }^{[6]}$ F. Effenberger, T. Kesmarszky, Chem. Ber. 1992, 125, $2103-2110$.

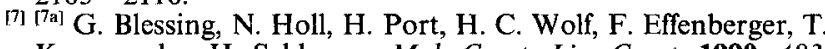
Kesmarszky, H. Schlosser, Mol. Cryst. Liq. Cryst. 1990, 183 , $21-30 .-{ }^{[7 b]}$ F. Effenberger, H. C. Wolf, New J. Chem. 1991, $15,117-123$

[8] [8a] P. Emele, Diplomarbeit, Univ. Stuttgart, 1990. - ${ }^{[8 b]}$ G. Blessing, Diplomarbeit, Univ. Stuttgart, 1989. - ${ }^{[8 c]}$ N. Holl, H. Strobel, F. Effenberger, H. Port, H. C. Wolf, Publikation in Vorbereitung.

[9] [9a] R. A. Keller, L. J. Dolby, J. Am. Chem. Soc. 1967, 89, $2768-2770 .{ }^{\left[{ }^{3 b}\right]}$ R. A. Keller, L. J. Dolby, J. Am. Chem. Soc. $1969,91,1293-1299$.

${ }^{[10]}$ Z.-Z. Wu, H. Morrison, J. Am. Chem. Soc, 1989, 111, $9267-9269$.

${ }^{[11]}$ Z. Tong, G. Yang, S. Wu, Chin. J. Chem. 1990, 61-67; Chem. Abstr. 1991, 114, 6925z.

[12] [12a] P. Pasman, N. W. Koper, J. W. Verhoeven, Recl. Trav. Chim. Pays-Bas 1982, 101, 363-364. - ${ }^{[12 b]}$ P. Pasman, G. F. Mes, N. W. Koper, J. W. Verhoeven, J. Am. Chem. Soc. 1985, 107, $5839-5843$.

[13] [13a] J. R. Miller, L. T. Calcaterra, G. L. Closs, J. Am. Chem. Soc. 1984, 106, 3047-3049. - ${ }^{[13 \mathrm{~b}]}$ M. D. Johnson, J. R. Miller, N S. Green, G. L. Closs, J. Phys. Chem. 1989, 93, 1173-1176. 
${ }^{[14]}$ A. Marquet, H. B. Kagan, U. Dvolaitzky, J. Lematre, J. Jacques, Bull. Soc. Chim. Fr. 1960, 539-547.

${ }^{[15]}$ W. Nagata, T. Wakabayashi, Y. Hayase, Org. Synth. 1973, 53, $44-48$.

${ }^{[16]}$ S. Akiyama, K. Nakasuji, M. Nakagawa, Bull. Chem. Soc. Jpn. 1971, 44, 2231-2236.

${ }^{[17]}$ W. G. Dauben, H. L. Bradlow, J. Am. Chem. Soc. 1952, 74, $559-560$.

${ }^{[18]}$ Y. Takeuchi, S. Akiyama, M. Nakagawa, Bull. Chem. Soc. Jpn. 1972, 45, 3183-3187.

${ }^{[19]}$ F. Effenberger, H. Schlosser, Synthesis 1990, 1085-1094.

${ }^{[20]}{ }^{200]}$ R. F. Hudson, P. A. Chopard, Helv. Chim. Acta 1962, 45 , 1137-1146. - ${ }^{[20 \mathrm{~b}]}$ R. F. Hudson, M. Green, Angew. Chem 1963, 75, 47-56; Angew. Chem. Int. Ed. Engl. 1963, 2, 11-20. $-{ }^{[20 \mathrm{Cc}]}$ C. T. Eyles, S. Trippett, J. Chem. Soc. 1966, 67-71. -
[200] E. H. Axelrod, G. M. Milne, E. E. van Tamelen, J. Am. Chem. Soc. 1970, 92, 2139-2141.

[21] [21a] W. S. Johnson, W. A. Vredenburgh, J. E. Pike, J. Am. Chem Soc. 1960, 82, 3409-3415. - ${ }^{[21 \mathrm{~b}]}$ C. Djerassi, R. R. Engle, A. Bowers, J. Org. Chem. 1956, 21, 1547-1549.

${ }^{[22]}{ }^{[22 a]}$ G. V. Ponomarev, G. B. Maravin, Chem. Heterocycl. Compd. 1982, 18, 50-55. - ${ }^{[22 b]}$ M. J. Crossley, M. M. Harding, S. Sternhell, J. Am. Chem. Soc. 1986, 108, 3608-3613.

${ }^{[23]} \mathrm{J}$. Wolinsky, K. L. Erickson, J. Org. Chem. 1965, 30, $2208-2211$. ${ }^{[24]}$ M. Momenteau, B. Loock, E. Bisagni, M. Rougee, Can. J. Chem. 1979, 57, 1804-1813.

${ }^{[25]}$ G. B. Maravin, G. V. Ponomarev, A. M. Shul'ga, Chem. Heterocycl. Compd. 1987, 23, 179-185.

${ }^{[26]}$ F. Effenberger, C.-P. Niesert, Synthesis 1992, 1137-1144.

[31/93] 